ESAIM: PROCEEDINGS AND SURVEYS, June 2017, Vol. 56, p. 88-110

S. Crépey, M. Jeanblanc and A. Nikeghbali Editors

\title{
SOME EXISTENCE RESULTS FOR ADVANCED BACKWARD STOCHASTIC DIFFERENTIAL EQUATIONS WITH A JUMP TIME*,**
}

\author{
Monique JEANBlAnC ${ }^{1}$, Thomas LIM $^{2}$ And Nacira AGRAM ${ }^{3}$
}

\begin{abstract}
In this paper, we are interested by advanced backward stochastic differential equations (ABSDEs), in a probability space equipped with a Brownian motion and a single jump process, with a jump at time $\tau$. ABSDEs are BSDEs where the driver depends on the future paths of the solution. We show, that under immersion hypothesis between the Brownian filtration and its progressive enlargement with $\tau$, assuming that the conditional law of $\tau$ is equivalent to the unconditional law of $\tau$, and a Lipschitz condition on the driver, the ABSDE has a solution.
\end{abstract}

\section{INTRODUCTION}

In the traditional approach, Backward Stochastic Differential Equations (BSDEs) are studied for a driver which depends in a Markovian way of the parameters; in particular, BSDEs with a single jump and driven by a Brownian motion $B$ have the form

$$
-d Y_{t}=f\left(t, Y_{t}, Z_{t}, U_{t}\right) d t-Z_{t} d B_{t}-U_{t} d H_{t}
$$

where $H$ is the process $H_{t}=\mathbb{1}_{\{\tau \leq t\}}$ associated with a given random time $\tau$. In this paper, we are interested by BSDEs in which the driver depends on the future of the solution, in such a way that the driver is adapted, for example if the driver depends on conditional expectation of the future of the solution, e.g., $-d Y_{t}=\left(a Y_{t}+\right.$ $\left.b \mathbb{E}^{\mathcal{G}_{t}}\left(Y_{t+1}\right)\right) d t-Z_{t} d W_{t}-U_{t} d H_{t}$, we use the notation $\mathbb{E}^{\mathcal{G}_{t}}[X]:=\mathbb{E}\left[X \mid \mathcal{G}_{t}\right]$, where $\mathbb{G}=\left(\mathcal{G}_{t}\right)_{t \geq 0}$ is the progressive enlargement filtration of the filtration of $B$ by $H$. More precisely, we focus on BSDEs written in one of the following forms, called advanced backward stochastic differential equations (in short ABSDEs)

$$
\left\{\begin{aligned}
-d Y_{t}= & f\left(t, Y_{t}, \mathbb{E}^{\mathcal{G}_{t}}\left[Y_{t+\delta}\right],\left(\mathbb{E}^{\mathcal{G}_{t}}\left[Y_{t+s}\right]\right)_{0 \leq s \leq \delta}, Z_{t}, \mathbb{E}^{\mathcal{G}_{t}}\left[Z_{t+\delta}\right],\left(\mathbb{E}^{\mathcal{G}_{t}}\left[Z_{t+s}\right]\right)_{0 \leq s \leq \delta},\right. \\
& \left.U_{t}, \mathbb{E}^{\mathcal{G}_{t}}\left[U_{t+\delta}\right],\left(\mathbb{E}^{\mathcal{G}_{t}}\left[U_{t+s}\right]\right)_{0 \leq s \leq \delta}\right) d t-Z_{t} d B_{t}-U_{t} d H_{t}, \quad 0 \leq t \leq T, \\
Y_{T+t}= & \xi_{T+t}, \quad 0 \leq t \leq \delta \\
Z_{T+t}= & P_{T+t}, \quad U_{T+t}=Q_{T+t} \mathbb{1}_{\{T+t \leq \tau\}}, \quad 0<t \leq \delta
\end{aligned}\right.
$$

* This research was supported by Chaire Markets in Transition, (French Banking Federation) Institut Louis Bachelier and Labex ANR 11-LABX-0019.

** The research of $N$. Agram is also carried out with support of the Norwegian Research Council, within the research project Challenges in Stochastic Control, Information and Applications (STOCONINF), project number 250768/F20.

${ }^{1}$ LaMME, University of Evry; monique.jeanblanc@univ-evry.fr

${ }^{2}$ LaMME, Ecole Nationale Supérieure d'Informatique pour l'Industrie et l'Entreprise; lim@ensiie.fr

${ }^{3}$ Department of Mathematics, University of Oslo; naciraa@math.uio.no

(C) EDP Sciences, SMAI 2017 
and

$$
\left\{\begin{aligned}
-d Y_{t}= & \mathbb{E}^{\mathcal{G}_{t}}\left[f \left(t, Y_{t}, Y_{t+\delta},\left(Y_{t+s}\right)_{0 \leq s \leq \delta}, Z_{t}, Z_{t+\delta},\left(Z_{t+s}\right)_{0 \leq s \leq \delta},\right.\right. \\
& \left.\left.U_{t}, U_{t+\delta},\left(U_{t+s}\right)_{0 \leq s \leq \delta}\right)\right] d t-Z_{t} d B_{t}-U_{t} d H_{t}, \quad 0 \leq t \leq T, \\
Y_{T+t}= & \xi_{T+t}, \quad 0 \leq t \leq \delta, \\
Z_{T+t}= & P_{T+t}, \quad U_{T+t}=Q_{T+t} \mathbb{1}_{\{T+t \leq \tau\}}, \quad 0<t \leq \delta .
\end{aligned}\right.
$$

The solution is the triplet $(Y, Z, U)$, living in some spaces we shall define later on. In this equation, for a process $V$, the notation $\left(V_{t+s}\right)_{0 \leq s \leq \delta}$ means that we consider all the path of the process between $t$ and $t+\delta$. The terminal conditions $\xi, P$ and $Q$ are given processes. We remark that the drivers $f$ of these ABSDEs depend on the values of the processes $(Y, Z, U)$ for present time $t$ as well as for future time $t+\delta$ and also of the trajectory of the processes on the interval $[t, t+\delta]$. The ABSDE (0.1) was introduced by Peng and Yang in [12] in a Brownian case setting (roughly speaking, for $\tau \equiv 0$ ). Øksendal et al. [11] have introduced ABSDEs of the form $(0.2)$ - in a simpler case where not all the path of the solution is involved - when dealing with optimal control for delayed systems, taking into account a random Poisson measure, instead of a single jump process.

Using the methodology of BSDEs in an enlargement of filtration setting as in Kharroubi and Lim [8], we give conditions such that there exists a unique solution of (0.1) and of (0.2) under immersion hypothesis and in adequate spaces. This progressive enlargement is often considered as progressive adding of information given in form of a random time $\tau$ in a way which transforms $\tau$ to a stopping time with respect to the filtration $\mathbb{G}$. The topic of enlargement of filtration was initiated by Jacod, Jeulin and Yor (see [6,7]). Naturally, the enlargement of filtration appears in credit risk and it has also been related recently to stochastic optimal control by Pham [13] and to mean-variance hedging by Kharroubi et al. [9] where the optimal strategy is described by non-standard BSDEs driven by a Brownian motion and a jump martingale in the enlarged filtration.

There are, in the literature, two approaches of BSDEs with a single jump. One of them considers BSDEs driven by martingales and is based on the predictable representation property (PRP), as in Dumitrescu et al. [3]. These authors consider a Brownian motion $B$ and the martingale $M$ associated to the jump process $H$. Under some conditions, in particular that the Brownian motion is a Brownian motion in the filtration generated by the pair $(B, M)$ (which is a consequence of the assumed immersion property), they prove that the pair $(B, M)$ enjoys PRP and they solve BSDEs of the form $-d Y_{t}=f\left(t, Y_{t}, Z_{t}, U_{t}\right) d t-Z_{t} d W_{t}-U_{t} d M_{t}$ using usual methodology. Kharroubi and Lim's method is different: they consider the case $-d Y_{t}=g\left(t, Y_{t}, Z_{t}, U_{t}\right) d t-Z_{t} d W_{t}-U_{t} d H_{t}$, assuming immersion property, and they show that this BSDE is equivalent to a system of BSDE in the Brownian filtration. In fact, assuming the more general hypothesis of existence of positive conditional density $\alpha$ (as in Hypothesis 1.2 below), they can solve the problem without immersion property as follows: The change of probability $d \mathbb{P}^{*}=\left(\alpha_{t}(\tau)\right)^{-1} d \mathbb{P}$ is such that $B$ is a $\mathbb{P}^{*}$-Brownian motion independent of $H$ so that immersion holds under $\mathbb{P}^{*}$ and the solution of the BSDE is the same under $\mathbb{P}$ and $\mathbb{P}^{*}$. However, here, we can not use this methodology, since a change of probability will affect the conditional expectation, and the Lipschitz condition will be difficult to check. Nevertheless, working without immersion hypothesis is doable as we explain in the Appendix of the paper.

\section{FRAMEWORK}

\subsection{Classical results about progressive enlargement}

Let $(\Omega, \mathcal{G}, \mathbb{P})$ be a complete probability space. We assume that this space is equipped with a one-dimensional standard Brownian motion $B$ and we denote by $\mathbb{F}:=\left(\mathcal{F}_{t}\right)_{t \geq 0}$ the right-continuous and complete filtration generated by $B$. We consider on this space a random time $\tau$ and we introduce the right-continuous process $H:=\mathbb{1}_{\{\tau \leq .\}}$. Since $\tau$ is not supposed to be an $\mathbb{F}$-stopping time, we use the standard approach of filtration enlargement by considering the smallest right-continuous extension $\mathbb{G}$ of $\mathbb{F}$ that turns $\tau$ into a $\mathbb{G}$-stopping time. More precisely, the filtration $\mathbb{G}:=\left(\mathcal{G}_{t}\right)_{t \geq 0}$ is defined by

$$
\mathcal{G}_{t}:=\bigcap_{\varepsilon>0} \tilde{\mathcal{G}}_{t+\varepsilon}
$$


for any $t \geq 0$, where $\tilde{\mathcal{G}}_{s}:=\mathcal{F}_{s} \vee \sigma\left(H_{u}, u \in[0, s]\right)$, for any $s \geq 0$.

We denote by $\mathcal{P}(\mathbb{F})$ (resp. $\mathcal{P}(\mathbb{G})$ ) the $\sigma$-algebra of $\mathbb{F}$ (resp. $\mathbb{G}$ )-predictable subsets of $\Omega \times \mathbb{R}_{+}$, i.e., the $\sigma$ algebra generated by the left-continuous $\mathbb{F}$ (resp. $\mathbb{G}$ )-adapted processes. We denote by $\mathcal{O}(\mathbb{F})$ (resp. $\mathcal{O}(\mathbb{G})$ ) the $\sigma$-algebra of $\mathbb{F}$ (resp. $\mathbb{G}$ )-optional subsets of $\Omega \times \mathbb{R}_{+}$, i.e., the $\sigma$-algebra generated by the right-continuous $\mathbb{F}$ (resp. $\mathbb{G}$ )-adapted processes.

We impose the following hypothesis introduced by Brémaud and Yor [2], which is classical in the filtration enlargement theory and is called $(\mathcal{H})$-hypothesis or immersion property.

Hypothesis 1.1. The process $B$ remains a $\mathbb{G}$-Brownian motion.

We observe that, since the filtration $\mathbb{F}$ is generated by the Brownian motion $B$, Hypothesis 1.1 is equivalent to all $\mathbb{F}$-martingales are also $\mathbb{G}$-martingales. In particular, the stochastic integral $\int_{0}^{t} X_{s} d B_{s}$ is a well defined $\mathbb{G}$-local martingale for all $\mathcal{P}(\mathbb{G})$-measurable processes $X$ such that $\int_{0}^{t}\left|X_{s}\right|^{2} d s<\infty$ a.s., for all $t \geq 0$.

We also introduce another hypothesis, often called the Jacod equivalence hypothesis (see, e.g., [1, chapter 4]), that the conditional law of $\tau$ is equivalent to the law of $\tau$ and that $\tau$ admits a density w.r.t. Lebesgue's measure, which will allow us to compute conditional expectations w.r.t. $\mathbb{G}$ in terms of conditional expectations w.r.t. $\mathbb{F}$.

Hypothesis 1.2. We assume that there exists a strictly positive $\mathcal{P}(\mathbb{F}) \otimes \mathcal{B}(\mathbb{R})$-measurable function $(\omega, t, u) \rightarrow$ $\alpha_{t}(\omega, u)$ continuous in $t$ such that

a) for any $\theta \geq 0$, the process $\left(\alpha_{t}(\theta)\right)_{t \geq 0}$ is an $\mathbb{F}$-martingale,

b) for any $t \geq 0$, the measure $\alpha_{t}(\omega, \theta) d \theta$ is a version of $\mathbb{P}\left(\tau \in d \theta \mid \mathcal{F}_{t}\right)(\omega)$, that is for any Borel function $f$ such that $f(\tau)$ is integrable, one has

$$
\mathbb{E}\left[f(\tau) \mid \mathcal{F}_{t}\right]=\int_{0}^{\infty} f(\theta) \alpha_{t}(\theta) d \theta, \quad \text { a.s. }
$$

In particular, the density of $\tau$ is $\alpha_{0}$.

In all the paper, Hypotheses 1.1 and 1.2 are in force.

We now recall some standard results that will be important for our purpose and we refer to [4] for their proofs.

We introduce the $\mathbb{F}$-supermartingale $G$ (called Azéma's supermartingale) defined as

$$
G_{t}:=\mathbb{P}\left(\tau>t \mid \mathcal{F}_{t}\right)=\int_{t}^{\infty} \alpha_{t}(\theta) d \theta, \quad t \geq 0 .
$$

The supermartingale $G$ is strictly positive, non-increasing and continuous. The process $M$ defined by

$$
M_{t}:=H_{t}-\int_{0}^{t \wedge \tau} \frac{\alpha_{s}(s)}{G_{s}} d s, \quad t \geq 0
$$

is a $\mathbb{G}$-martingale, with a single jump at time $\tau$. The $\mathbb{F}$-adapted process $\lambda$ defined by

$$
\lambda_{t}:=\frac{\alpha_{t}(t)}{G_{t}}, \quad t \geq 0,
$$

is called the $\mathbb{F}$-intensity of $\tau$. Under Hypotheses 1.1 and 1.2, we have, from [4, equality (11)],

$$
\alpha_{t}(\theta)=\alpha_{\theta}(\theta), \quad \forall t \geq \theta
$$


which implies

$$
G_{t}=\exp \left(-\int_{0}^{t} \lambda_{s} d s\right)
$$

since, by definition of $G$ and $\lambda$ and the fact that (1.2) holds, we have the following equalities,

$$
G_{t}=\int_{t}^{\infty} \alpha_{t}(\theta) d \theta=1-\int_{0}^{t} \alpha_{t}(\theta) d \theta=1-\int_{0}^{t} \alpha_{\theta}(\theta) d \theta=1-\int_{0}^{t} G_{\theta} \lambda_{\theta} d \theta
$$

and $G_{0}=1$. Note that, from immersion

$$
G_{t}=\mathbb{E}^{\mathcal{F}_{s}}\left(\mathbb{1}_{\{\tau>t\}}\right), \quad \forall s>t .
$$

Hypothesis 1.3. We assume that the process $\lambda$ is upper bounded by a constant $k$.

Lemma 1.4. For any $t \in[0, T]$, the random variable $G_{t}$ is lower bounded by $e^{-k t}$, and for any $\theta \in[0, T]$ we have $0<\alpha_{t}(\theta) \leq k$.

Proof. The bound on $G$ is obvious from (1.3). Let $\theta \in[0, T]$, then for any $t \geq \theta,(1.1)$ and (1.2) lead to

$$
\alpha_{t}(\theta)=\alpha_{\theta}(\theta)=\lambda_{\theta} G_{\theta} \leq k .
$$

Moreover, since $\alpha(\theta)$ is a martingale, we get $\alpha_{t}(\theta) \leq \mathbb{E}^{\mathcal{F}_{t}}\left(\alpha_{\theta}(\theta)\right) \leq k$ for any $t \leq \theta$.

Furthermore, if $Y \in \mathcal{G}_{T}$ is integrable, then we have

$$
\mathbb{E}^{\mathcal{G}_{t}}\left[Y \mathbb{1}_{\{t<\tau\}}\right]=\frac{1}{G_{t}} \mathbb{1}_{\{t<\tau\}} \mathbb{E}^{\mathcal{F}_{t}}\left(Y \mathbb{1}_{\{t<\tau\}}\right)
$$

We recall a decomposition result for $\mathcal{P}(\mathbb{G})$-measurable processes, proved in [7, Lemma 4.4] for bounded processes. It can be easily extended to the case of unbounded processes.

Proposition 1.5. Any $\mathcal{P}(\mathbb{G})$-measurable process $X=\left(X_{t}\right)_{t \geq 0}$ can be represented as

$$
X_{t}=X_{t}^{b} \mathbb{1}_{\{t \leq \tau\}}+X_{t}^{a}(\tau) \mathbb{1}_{\{t>\tau\}},
$$

for all $t \geq 0$, where $X^{b}$ is $\mathcal{P}(\mathbb{F})$-measurable and $X^{a}(\cdot)$ is $\mathcal{P}(\mathbb{F}) \otimes \mathcal{B}\left(\mathbb{R}_{+}\right)$-measurable.

Here, the superscript $b$ is for before $\tau$ and $a$ for after $\tau$. In particular, a $\mathbb{G}$-predictable process is equal to an $\mathbb{F}$-predictable process on the set $\{t \leq \tau\}$.

Song [14] has extended the previous result to the class of optional processes under some hypotheses, which are satisfied under equivalence Jacod's hypothesis.

Proposition 1.6. Any $\mathcal{O}(\mathbb{G})$-measurable process $X=\left(X_{t}\right)_{t \geq 0}$ can be represented as

$$
X_{t}=X_{t}^{b} \mathbb{1}_{\{t<\tau\}}+X_{t}^{a}(\tau) \mathbb{1}_{\{t \geq \tau\}},
$$

for all $t \geq 0$, where $X^{b}$ is $\mathcal{O}(\mathbb{F})$-measurable and $X^{a}(\cdot)$ is $\mathcal{O}(\mathbb{F}) \otimes \mathcal{B}\left(\mathbb{R}_{+}\right)$-measurable.

If the process $X$ is bounded by a constant $K$, then the process $X^{b}$ is bounded by $K$ and one can also choose the process $X^{a}(\theta)$ bounded by $K$ for any $\theta \geq 0$. We remark that the uniqueness of $X_{t}^{a}(\theta)$ is granted for $\theta \leq t$.

The process $X^{b}$ is uniquely determined on $[0, T]$ by $X_{t}^{b}=\frac{1}{G_{t}} \mathbb{E}^{\mathcal{F}_{t}}\left[X_{t} \mathbb{1}_{\{t<\tau\}}\right]$, this quantity will be called the pre-default part. 
Lemma 1.7. Let $Y_{T}(\tau)$ be a bounded $\mathcal{F}_{T} \otimes \sigma(\tau)$-measurable random variable. Then, for any $t \leq T$, we have

$$
\mathbb{E}^{\mathcal{G}_{t}}\left[Y_{T}(\tau)\right]=Y_{t}^{b} \mathbb{1}_{\{t<\tau\}}+Y_{t}^{a}(\tau) \mathbb{1}_{\{\tau \leq t\}} \quad \text { a.s. }
$$

where

$$
\begin{aligned}
Y_{t}^{b} & =\frac{\mathbb{E}^{\mathcal{F}_{t}}\left[\int_{t}^{\infty} Y_{T}(u) \alpha_{T}(u) d u\right]}{G_{t}} \text { a.s. } \\
Y_{t}^{a}(\theta) & =\mathbb{E}^{\mathcal{F}_{t}}\left[Y_{T}(\theta)\right] \text { a.s. for any } \theta \leq t
\end{aligned}
$$

which can be rewritten under the form

$$
Y_{t}^{a}(\tau)=\mathbb{E}^{\mathcal{F}_{t}}\left[Y_{T}(\theta)\right]_{\mid \theta=\tau} \quad \text { a.s. on } \tau \leq t
$$

Proof. The proof of this lemma is an application of Proposition 1.6 and that

$$
Y_{t}^{a}(\theta)=\frac{\mathbb{E}^{\mathcal{F}_{t}}\left[Y_{T}(\theta) \alpha_{T}(\theta)\right]}{\alpha_{t}(\theta)} \text { a.s. }
$$

and $\alpha_{t}(\theta)=\alpha_{\theta}(\theta)$ for any $t \geq \theta$.

Therefore, if $Y_{T}(\tau)$ is bounded by a constant $K$ then the processes $Y^{b}$ and $Y^{a}(\theta)$ are bounded by $K$ for any $\theta \geq 0$.

We now give a decomposition result for some Stochastic Differential Equations (SDEs) in $\mathbb{G}$ in terms of SDEs in $\mathbb{F}$.

Lemma 1.8. If the process $X$ satisfies the following stochastic differential equation

$$
d X_{t}=\mu\left(t, X_{t}, \eta_{t}\right) d t+\sigma\left(t, X_{t}, \eta_{t}\right) d B_{t}+\varphi\left(t, X_{t^{-}}, \eta_{t}\right) d H_{t}
$$

where $\mu, \sigma$ are $\mathcal{O}(\mathbb{G}) \otimes \mathcal{B}(\mathbb{R}) \otimes \mathcal{B}(\mathbb{R})$-measurable maps, $\varphi$ is a $\mathcal{P}(\mathbb{G}) \otimes \mathcal{B}(\mathbb{R}) \otimes \mathcal{B}(\mathbb{R})$ measurable map and $\eta$ is a $\mathbb{G}$-predictable process, then $X^{a}$ and $X^{b}$ satisfy

$$
\left\{\begin{aligned}
d X_{t}^{a}(\tau) & =\mu^{a}\left(t, \tau, X_{t}^{a}(\tau), \eta_{t}^{a}(\tau)\right) d t+\sigma^{a}\left(t, \tau, X_{t}^{a}(\tau), \eta_{t}^{a}(\tau)\right) d B_{t}, \quad \tau \leq t \leq T \\
d X_{t}^{b} & =\mu^{b}\left(t, X_{t}^{b}, \eta_{t}^{b}\right) d t+\sigma^{b}\left(t, X_{t}^{b}, \eta_{t}^{b}\right) d B_{t}, \quad 0 \leq t \leq T \\
X_{t}^{a}(t)-X_{t}^{b} & =\varphi\left(t, X_{t}^{b}, \eta_{t}^{b}\right), \quad 0 \leq t \leq \tau
\end{aligned}\right.
$$

Proof. The proof of this lemma is an application of Proposition 1.6 and for the last equality, we have used that if an $\mathbb{F}$-predictable process $K$ satisfies $K_{\tau}=0$, then $K_{t}=0$ on $\{t \leq \tau\}$ (see [10, Lemma 3, Chapter 1]).

\subsection{Notations}

To define solutions to ABSDEs, we introduce the following spaces, where $s, t \in \mathbb{R}_{+}$with $s \leq t$, and $T<\infty$ is the terminal time and $\delta$ is a strictly positive constant.

- $\mathcal{S}_{\mathbb{G}}^{2}[s, t]\left(\right.$ resp. $\left.\mathcal{S}_{\mathbb{F}}^{2}[s, t]\right)$ is the set of $\mathbb{R}$-valued $\mathcal{O}(\mathbb{G})($ resp. $\mathcal{O}(\mathbb{F}))$-measurable processes $\left(Y_{u}\right)_{u \in[s, t]}$ such that

$$
\|Y\|_{\mathcal{S}^{2}[s, t]}^{2}:=\mathbb{E}\left[\sup _{u \in[s, t]}\left|Y_{u}\right|^{2}\right]<\infty
$$


- $L_{\mathbb{G}}^{2}[s, t]\left(\right.$ resp. $\left.L_{\mathbb{F}}^{2}[s, t]\right)$ is the set of $\mathbb{R}$-valued $\mathcal{P}(\mathbb{G})\left(\right.$ resp. $\mathcal{P}(\mathbb{F})$ )-measurable processes $\left(Z_{u}\right)_{u \in[s, t]}$ such that

$$
\|Z\|_{L^{2}[s, t]}^{2}:=\mathbb{E}\left[\int_{s}^{t}\left|Z_{u}\right|^{2} d u\right]<\infty
$$

- $L^{2}\left(\mathcal{F}_{t}\right)$ is the set of $\mathbb{R}$-valued square integrable $\mathcal{F}_{t}$-measurable random variables.

- $L_{\tau}^{2}$ is the set of $\mathbb{R}$-valued $\mathcal{P}(\mathbb{F})$-measurable processes $U$ such that $U_{t}=0$ for $t>\tau$ and

$$
\|U\|_{L_{\tau}^{2}}^{2}:=\mathbb{E}\left[\int_{0}^{T}\left|U_{s}\right|^{2} d s\right]<\infty .
$$

- $\mathbb{D}[0, \delta]$ is the set of càd-làg $\mathbb{R}$-valued maps defined on $[0, \delta]$. For $\mathcal{Y} \in \mathbb{D}[0, \delta]$, we denote $|\mathcal{Y}|:=$ $\frac{1}{\sqrt{\delta}} \int_{0}^{\delta}|\mathcal{Y}(s)| d s$.

\subsection{Existence results for ABSDE in a Brownian filtration}

We extend the results of Peng and Yang [12] to more general drivers since we assume in our case that the driver depends on the trajectory of the processes on the interval $[t, t+\delta]$. The proofs are based on standard methodologies, however they require some care to check the needed Lipschitz conditions. To simplify the writing we introduce some new notations for each Proposition, and the same notation $\overrightarrow{\mathbf{y}} \in \mathbf{A}$ is used in different meanings which are clear from the context.

Proposition 1.9. Let $\mathbf{A}:=\mathbb{R}^{2} \times \mathbb{D}[0, \delta] \times \mathbb{R}^{2} \times \mathbb{D}[0, \delta]$ and, for any $\overrightarrow{\mathbf{y}}=(y, \widehat{y}, \mathcal{Y}, z, \widehat{z}, \mathcal{Z}) \in \mathbf{A}$ we define $|\overrightarrow{\mathbf{y}}|$ by

$$
|\overrightarrow{\mathbf{y}}|=|y|+|\widehat{y}|+|\mathcal{Y}|+|z|+|\widehat{z}|+|\mathcal{Z}|,
$$

where $|\mathcal{Y}|$ is defined in Section 1.2. Let $f$ be a map from $\Omega \times[0, T] \times \mathbf{A}$ valued in $\mathbb{R}$. Let $p$ and $q$ be given bounded $\mathbb{F}$-adapted processes.

The following ABSDE

$$
\left\{\begin{aligned}
-d Y_{t}= & f\left(t, Y_{t}, \mathbb{E}^{\mathcal{F}_{t}}\left[p_{t+\delta} Y_{t+\delta}\right],\left\{\mathbb{E}^{\mathcal{F}_{t}}\left[p_{t+s} Y_{t+s}\right]\right\}_{0 \leq s \leq \delta}, Z_{t}, \mathbb{E}^{\mathcal{F}_{t}}\left[q_{t+\delta} Z_{t+\delta}\right],\left\{\mathbb{E}^{\mathcal{F}_{t}}\left[q_{t+s} Z_{t+s}\right]\right\}_{0 \leq s \leq \delta}\right) d t \\
& -Z_{t} d B_{t}, \quad 0 \leq t \leq T \\
Y_{T+t}= & \xi_{T+t}, \quad 0 \leq t \leq \delta \\
Z_{T+t}= & P_{T+t}, \quad 0<t \leq \delta
\end{aligned}\right.
$$

has a unique solution in $\mathcal{S}_{\mathbb{F}}^{2}[0, T+\delta] \times L_{\mathbb{F}}^{2}[0, T+\delta]$ if

a) the map $f(\cdot, \overrightarrow{\mathbf{y}})$ is optional for any $\overrightarrow{\mathbf{y}} \in \mathbf{A}$,

b) there exists $C>0$ such that, for any $t \in[0, T]$, any $\overrightarrow{\mathbf{y}} \in \mathbf{A}$, we have

$$
\left|f(t, \overrightarrow{\mathbf{y}})-f\left(t, \overrightarrow{\mathbf{y}}^{\prime}\right)\right| \leq C\left|\overrightarrow{\mathbf{y}}-\overrightarrow{\mathbf{y}}^{\prime}\right|
$$

c) $\mathbb{E}\left[\int_{0}^{T}|f(s, \overrightarrow{\mathbf{0}})|^{2} d s\right]<\infty$,

d) the terminal condition $\xi$ belongs to $\mathcal{S}_{\mathbb{F}}^{2}[T, T+\delta]$ and $P$ belongs to $L_{\mathbb{F}}^{2}[T, T+\delta]$.

Proof. In the driver, the map $\mathcal{Y}=\left(\mathcal{Y}_{t}(s)=\mathbb{E}^{\mathcal{F}_{t}}\left[p_{t+s} Y_{t+s}\right], 0 \leq s \leq \delta\right)$ is a family of $\mathcal{F}_{t}$-measurable random variables. Let us first introduce a norm in the Banach space $E:=\mathcal{S}_{\mathbb{F}}^{2}[0, T+\delta] \times L_{\mathbb{F}}^{2}[0, T+\delta]$ for $\beta>0$ : for $(Y, Z) \in E$

$$
\|(Y, Z)\|_{\beta}^{2}:=\mathbb{E}\left[\int_{0}^{T+\delta} e^{\beta t}\left(Y_{t}^{2}+Z_{t}^{2}\right) d t\right]
$$


and define the mapping $\Phi: E \rightarrow E$ by $\Phi((y, z))=(Y, Z)$ where $(Y, Z)$ is defined by

$$
\left\{\begin{array}{l}
-d Y_{t}=f\left(t, \overrightarrow{\mathbf{y}}_{t}\right) d t-Z_{t} d B_{t}, \quad 0 \leq t \leq T \\
Y_{T+t}=\xi_{T+t}, \quad 0 \leq t \leq \delta \\
Z_{T+t}=P_{T+t}, \quad 0<t \leq \delta
\end{array}\right.
$$

where $\overrightarrow{\mathbf{y}}_{t}=\left(y_{t}, \mathbb{E}^{\mathcal{F}_{t}}\left[p_{t+\delta} y_{t+\delta}\right],\left\{\mathbb{E}^{\mathcal{F}_{t}}\left[p_{t+s} y_{t+s}\right]\right\}_{0 \leq s \leq \delta}, z_{t}, \mathbb{E}^{\mathcal{F}_{t}}\left[q_{t+\delta} z_{t+\delta}\right],\left\{\mathbb{E}^{\mathcal{F}_{t}}\left[q_{t+s} z_{t+s}\right]\right\}_{0 \leq s \leq \delta}\right)$. We now prove that $\Phi$ is a contraction in $E$ under the norm $\|.\|_{\beta}$. For two arbitrary elements $(y, z)$ and $\left(y^{\prime}, z^{\prime}\right)$, we denote their difference by

$$
(\widetilde{y}, \widetilde{z})=\left(y-y^{\prime}, z-z^{\prime}\right) .
$$

We can prove by using classical estimates that we have

$$
\mathbb{E}\left[\int_{0}^{T} e^{\beta t}\left(\frac{\beta}{2} \widetilde{Y}_{t}^{2}+\widetilde{Z}_{t}^{2}\right) d t\right] \leq \frac{2}{\beta} \mathbb{E}\left[\int_{0}^{T} e^{\beta t}\left|f\left(t, \overrightarrow{\mathbf{y}}_{t}\right)-f\left(t, \overrightarrow{\mathbf{y}}_{t}^{\prime}\right)\right|^{2} d t\right]
$$

In the following inequalities, $K$ is a constant which does not depend on $\beta$ and may change from line to line. By Lipschitz property of the map $f$, the fact that the square of a sum (resp. integral) is bounded by the sum (resp. integral) of the square (up to a constant) and the boundedness of $p$ and $q$, it follows that

$$
\mathbb{E}\left[\int_{0}^{T} e^{\beta t}\left(\frac{\beta}{2} \widetilde{Y}_{t}^{2}+\widetilde{Z}_{t}^{2}\right) d t\right] \leq \frac{K}{\beta} \mathbb{E}\left[\int_{0}^{T} e^{\beta t}\left(\widetilde{y}_{t}^{2}+\widetilde{z}_{t}^{2}+\widetilde{y}_{t+\delta}^{2}+\widetilde{z}_{t+\delta}^{2}+\frac{1}{\delta} \int_{0}^{\delta}\left(\widetilde{y}_{t+s}^{2}+\widetilde{z}_{t+s}^{2}\right) d s\right) d t\right]
$$

By the change of variable $u=t+s$, we get

$$
\mathbb{E}\left[\int_{0}^{T} e^{\beta t}\left(\frac{\beta}{2} \widetilde{Y}_{t}^{2}+\widetilde{Z}_{t}^{2}\right) d t\right] \leq \frac{K}{\beta} \mathbb{E}\left[\int_{0}^{T+\delta} e^{\beta t}\left(\widetilde{y}_{t}^{2}+\widetilde{z}_{t}^{2}\right) d t+\frac{1}{\delta} \int_{0}^{T} e^{\beta t} \int_{t}^{t+\delta}\left(\widetilde{y}_{u}^{2}+\widetilde{z}_{u}^{2}\right) d u d t\right] .
$$

Fubini's theorem leads to

$$
\begin{aligned}
\frac{1}{\delta} \int_{0}^{T} e^{\beta t} \int_{t}^{t+\delta}\left(\widetilde{y}_{u}^{2}+\widetilde{z}_{u}^{2}\right) d u d t & \leq \frac{1}{\delta} \int_{0}^{T+\delta}\left(\int_{u-\delta}^{u} e^{\beta t} d t\right)\left(\widetilde{y}_{u}^{2}+\widetilde{z}_{u}^{2}\right) d u \\
& \leq \frac{\left(1-e^{-\beta \delta}\right)}{\beta \delta} \int_{0}^{T+\delta} e^{\beta u}\left(\widetilde{y}_{u}^{2}+\widetilde{z}_{u}^{2}\right) d u \\
& \leq \int_{0}^{T+\delta} e^{\beta u}\left(\widetilde{y}_{u}^{2}+\widetilde{z}_{u}^{2}\right) d u
\end{aligned}
$$

where we have used that $1-e^{-\beta \delta} \leq \beta \delta$.

Combining (1.10) with (1.9), we obtain for $\beta \geq 2$

$$
\mathbb{E}\left[\int_{0}^{T} e^{\beta t}\left(\widetilde{Y}_{t}^{2}+\widetilde{Z}_{t}^{2}\right) d t\right] \leq \frac{K}{\beta} \mathbb{E}\left[\int_{0}^{T+\delta} e^{\beta t}\left(\widetilde{y}_{t}^{2}+\widetilde{z}_{t}^{2}\right) d t\right] .
$$

Consequently, since $\widetilde{Y}=\widetilde{Z}=0$ for $t>T$, we get

$$
\|(\widetilde{Y}, \widetilde{Z})\|_{\beta}^{2} \leq \frac{K}{\beta}\|(\widetilde{y}, \widetilde{z})\|_{\beta}^{2},
$$


and $\Phi$ is a contraction on $\mathcal{S}_{\mathbb{F}}^{2}[0, T+\delta] \times L_{\mathbb{F}}^{2}[0, T+\delta]$ for $\beta$ large enough to ensure that $K / \beta<1$, and $\beta>2$. As $\Phi$ is a contraction, using general results on $\operatorname{BSDE}$ (as in [5]) there exists a unique solution $(Y, Z$ ) in $\mathcal{S}_{\mathbb{F}}^{2}[0, T+\delta] \times L_{\mathbb{F}}^{2}[0, T+\delta]$ to $\operatorname{ABSDE}(1.7)$.

We now give an estimation of the solution of the ABSDE.

Proposition 1.10. Suppose $f$ satisfies the hypotheses of Proposition 1.9. Then there exists a strictly positive constant $K$ that only depends on the Lipschitz constant $C$ and on $T$ such that for any $\xi \in \mathcal{S}_{\mathbb{F}}^{2}[T, T+\delta]$ and $P \in L_{\mathbb{F}}^{2}[T, T+\delta]$, the solution $(Y, Z)$ of the ABSDE (1.7) satisfies

$$
\mathbb{E}^{\mathcal{F}_{t}}\left[\sup _{t \leq s \leq T} Y_{s}^{2}+\int_{t}^{T} Z_{s}^{2} d s\right] \leq K \mathbb{E}^{\mathcal{F}_{t}}\left[\xi_{T}^{2}+\int_{T}^{T+\delta}\left(\xi_{s}^{2}+P_{s}^{2}\right) d s+\int_{t}^{T} f(s, \overrightarrow{\mathbf{0}})^{2} d s\right]
$$

for any $t \in[0, T]$.

Proof. The proof is obtained with standard computations. For the sake of completeness, we give details in the Appendix.

Using the same methodology as in Proposition 1.9 , one obtains the following result, where $\mathbb{D}(t,[0, \delta])$ is the family of maps $\mathcal{Y}$ from $[0, \delta]$ to $\mathbb{R}$ such that $\mathcal{Y}(s)$ is $\mathcal{F}_{t+s}$-measurable, for any $s \in[0, \delta]$.

Proposition 1.11. For any $t \in[0, T]$, let $\mathbf{A}_{t}=\mathbb{R} \times L^{2}\left(\mathcal{F}_{t+\delta}\right) \times \mathbb{D}(t,[0, \delta]) \times \mathbb{R} \times L^{2}\left(\mathcal{F}_{t+\delta}\right) \times \mathbb{D}(t,[0, \delta])$ and for any $\overrightarrow{\mathbf{y}}=(y, \zeta, \mathcal{Y}, z, \eta, \mathcal{Z}) \in \mathbf{A}_{t}$, we introduce $|\overrightarrow{\mathbf{y}}|=|y|+|z|+\mathbb{E}^{\mathcal{F}_{t}}(|\zeta|+|\eta|+|\mathcal{Y}|+|\mathcal{Z}|)$. For a map $f$ such that $f(\omega, t): \mathbf{A}_{t} \rightarrow \mathbb{R}$, the following ABSDE

$$
\left\{\begin{array}{l}
-d Y_{t}=f\left(t, Y_{t}, Y_{t+\delta},\left(Y_{t+s}\right)_{0 \leq s \leq \delta}, Z_{t}, Z_{t+\delta},\left(Z_{t+s}\right)_{0 \leq s \leq \delta}\right) d t-Z_{t} d B_{t}, \quad 0 \leq t \leq T \\
Y_{T+t}=\xi_{T+t}, \quad 0 \leq t \leq \delta \\
Z_{T+t}=P_{T+t}, \quad 0<t \leq \delta
\end{array}\right.
$$

has a unique solution in $\mathcal{S}_{\mathbb{F}}^{2}[0, T+\delta] \times L_{\mathbb{F}}^{2}[0, T+\delta]$ if the map $f$ satisfies:

a) for $\overrightarrow{\mathbf{y}}_{t} \in \mathbf{A}_{t}, f\left(t, \overrightarrow{\mathbf{y}}_{t}\right)$ is $\mathcal{F}_{t}$-measurable,

b) there exists $C$ such that for any $t \in[0, T]$, any $\overrightarrow{\mathbf{y}}, \overrightarrow{\mathbf{y}}^{\prime}$ in $\mathbf{A}_{t}$, one has

$$
\left|f(t, \overrightarrow{\mathbf{y}})-f\left(t, \overrightarrow{\mathbf{y}}^{\prime}\right)\right| \leq C\left|\overrightarrow{\mathbf{y}}-\overrightarrow{\mathbf{y}^{\prime}}\right|
$$

c) $\mathbb{E}\left(\int_{0}^{T}|f(t, \overrightarrow{\mathbf{0}})|^{2} d t\right)<\infty$,

d) the terminal condition $\xi$ belongs to $\mathcal{S}_{\mathbb{F}}^{2}[T, T+\delta]$ and $P$ belongs to $L_{\mathbb{F}}^{2}[T, T+\delta]$.

Moreover, there exists a constant $K$ such that we have

$$
\mathbb{E}^{\mathcal{F}_{t}}\left[\sup _{t \leq s \leq T} Y_{s}^{2}+\int_{t}^{T} Z_{s}^{2} d s\right] \leq K \mathbb{E}^{\mathcal{F}_{t}}\left[\xi_{T}^{2}+\int_{T}^{T+\delta}\left(\xi_{s}^{2}+P_{s}^{2}\right) d s+\int_{t}^{T} f(s, \overrightarrow{\mathbf{0}})^{2} d s\right]
$$

for any $t \in[0, T]$.

Proof. We use similar arguments to the proofs of Proposition 1.9 and 1.10. 


\section{ABSDE With JUMP OF TYPE (0.1)}

We assume that Hypotheses 1.1, 1.2 and 1.3 hold and that $f(., \overrightarrow{\mathbf{y}})$ is optional. We consider in this section an ABSDE of the following form: find a triple $(Y, Z, U) \in \mathcal{S}_{\mathbb{G}}^{2}[0, T+\delta] \times L_{\mathbb{G}}^{2}[0, T+\delta] \times L_{\tau}^{2}$ satisfying

$$
\left\{\begin{aligned}
-d Y_{t}= & f\left(t, Y_{t}, \mathbb{E}^{\mathcal{G}_{t}}\left[Y_{t+\delta}\right],\left\{\mathbb{E}^{\mathcal{G}_{t}}\left[Y_{t+s}\right]\right\}_{0 \leq s \leq \delta}, Z_{t}, \mathbb{E}^{\mathcal{G}_{t}}\left[Z_{t+\delta}\right],\left\{\mathbb{E}^{\mathcal{G}_{t}}\left[Z_{t+s}\right]\right\}_{0 \leq s \leq \delta},\right. \\
& \left.U_{t}, \mathbb{E}^{\mathcal{G}_{t}}\left[U_{t+\delta}\right],\left\{\mathbb{E}^{\mathcal{G}_{t}}\left[U_{t+s}\right]\right\}_{0 \leq s \leq \delta}\right) d t-Z_{t} d B_{t}-U_{t} d H_{t}, \quad 0 \leq t \leq T \\
Y_{T+t}= & \xi_{T+t}, \quad 0 \leq t \leq \delta \\
Z_{T+t}= & P_{T+t}, \quad U_{T+t}=Q_{T+t} \mathbb{1}_{\{T+t \leq \tau\}}, \quad 0<t \leq \delta
\end{aligned}\right.
$$

From Propositions 1.5 and 1.6, all the involved processes can be decomposed in two parts, before and after $\tau$. In particular, since $\xi$ will be given as a $\mathbb{G}$-optional process and $P$ as a $\mathbb{G}$-predictable process, we have for any $t \in[0, T]$

$$
f(t, \overrightarrow{\mathbf{y}})=f^{b}(t, \overrightarrow{\mathbf{y}}) \mathbb{1}_{\{t<\tau\}}+f^{a}(t, \tau, \overrightarrow{\mathbf{y}}) \mathbb{1}_{\{t \geq \tau\}} \quad \text { (optional decomposition) }
$$

and we have for any $t \in[T, T+\delta]$

$$
\left\{\begin{array}{c}
\xi_{t}=\xi_{t}^{b} \mathbb{1}_{\{t<\tau\}}+\xi_{t}^{a}(\tau) \mathbb{1}_{\{t \geq \tau\}} \quad \text { (optional decomposition) } \\
P_{t}=P_{t}^{b} \mathbb{1}_{\{t \leq \tau\}}+P_{t}^{a}(\tau) \mathbb{1}_{\{t>\tau\}} \quad \text { (predictable decomposition) } .
\end{array}\right.
$$

We work under the following hypotheses:

Hypotheses 2.1. Let $\mathbf{A}:=\mathbb{R}^{2} \times \mathbb{D}[0, \delta] \times \mathbb{R}^{2} \times \mathbb{D}[0, \delta] \times \mathbb{R}^{2} \times \mathbb{D}[0, \delta]$ and, for any $\overrightarrow{\mathbf{y}} \in \mathbf{A}$, we define $|\overrightarrow{\mathbf{y}}|$ by

$$
|\overrightarrow{\mathbf{y}}|=|y|+|\hat{y}|+|\mathcal{Y}|+|z|+|\hat{z}|+|\mathcal{Z}|+|u|+|\hat{u}|+|\mathcal{U}| .
$$

a) The terminal conditions satisfy $\xi \in \mathcal{S}_{\mathbb{G}}^{2}[T, T+\delta], P \in L_{\mathbb{G}}^{2}[T, T+\delta], Q \in L_{\mathbb{F}}^{2}[T, T+\delta]$, there exists a constant $K$ such that $\mathbb{E}\left[\left|\xi_{u}^{a}(\theta)\right|^{2}\right] \leq K$ and $\mathbb{E}\left[\left|P_{u}^{a}(\theta)\right|^{2}\right] \leq K$ for any $(\theta, u) \in[0, T] \times[T, T+\delta]$.

b) The driver $f: \Omega \times[0, T] \times \mathbf{A} \rightarrow \mathbb{R}$ of the ABSDE is Lipschitz, i.e., there exists a constant $C$ such that, for any $t \in[0, T]$, any $\overrightarrow{\mathbf{y}}$ and $\overrightarrow{\mathbf{y}}^{\prime}$ in $\mathbf{A}$, we have

$$
\left|f(t, \overrightarrow{\mathbf{y}})-f\left(t, \overrightarrow{\mathbf{y}}^{\prime}\right)\right| \leq C\left|\overrightarrow{\mathbf{y}}-\overrightarrow{\mathbf{y}}^{\prime}\right|
$$

c) For any $\overrightarrow{\mathbf{y}} \in \mathbf{A}$, the process $f(\cdot, \overrightarrow{\mathbf{y}})$ is $\mathbb{G}$-optional.

d) There exists a constant $C^{\prime}$ such that $|f(s, \overrightarrow{\mathbf{0}})|<C^{\prime}$.

From Propositions 1.5 and 1.6, we can write

$$
\begin{cases}Y_{t}=Y_{t}^{b} \mathbb{1}_{\{t<\tau\}}+Y_{t}^{a}(\tau) \mathbb{1}_{\{t \geq \tau\}} & \text { (optional decomposition) } \\ Z_{t}=Z_{t}^{b} \mathbb{1}_{\{t \leq \tau\}}+Z_{t}^{a}(\tau) \mathbb{1}_{\{t>\tau\}} & \text { (predictable decomposition) }\end{cases}
$$

It follows, from Lemma 1.8, that

$$
\left\{\begin{aligned}
-d Y_{t}^{a}(\tau)= & f^{a}\left(t, \tau, Y_{t}^{a}(\tau), \mathbb{E}^{\mathcal{G}_{t}}\left[Y_{t+\delta}^{a}(\tau)\right],\left\{\mathbb{E}^{\mathcal{G}_{t}}\left[Y_{t+s}^{a}(\tau)\right]\right\}_{0 \leq s \leq \delta}, Z_{t}^{a}(\tau), \mathbb{E}^{\mathcal{G}_{t}}\left[Z_{t+\delta}^{a}(\tau)\right],\right. \\
& \left.\left\{\mathbb{E}^{\mathcal{G}_{t}}\left[Z_{t+s}^{a}(\tau)\right]\right\}_{0 \leq s \leq \delta}, 0,0,0\right) d t-Z_{t}^{a}(\tau) d B_{t}, \quad T \wedge \tau \leq t \leq T \\
Y_{T+t}^{a}(\tau)= & \xi_{T+t}^{a}(\tau), \quad 0 \leq t \leq \delta \\
Z_{T+t}^{a}(\tau)= & P_{T+t}^{a}(\tau), \quad 0<t \leq \delta
\end{aligned}\right.
$$


and

$$
\left\{\begin{aligned}
-d Y_{t}^{b}= & f^{b}\left(t, Y_{t}^{b}, \mathbb{E}^{\mathcal{F}_{t}}\left[Y_{t+\delta}\right],\left\{\mathbb{E}^{\mathcal{F}_{t}}\left[Y_{t+s}\right]\right\}_{0 \leq s \leq \delta}, Z_{t}^{b}, \mathbb{E}^{\mathcal{F}_{t}}\left[Z_{t+\delta}\right],\left\{\mathbb{E}^{\mathcal{F}_{t}}\left[Z_{t+s}\right]\right\}_{0 \leq s \leq \delta},\right. \\
& \left.Y_{t}^{a}(t)-Y_{t}^{b}, \mathbb{E}^{\mathcal{F}_{t}}\left[U_{t+\delta}\right],\left\{\mathbb{E}^{\mathcal{F}_{t}}\left[U_{t+s}\right]\right\}_{0 \leq s \leq \delta}\right) d t-Z_{t}^{b} d B_{t}, \quad 0 \leq t \leq T, \\
Y_{T+t}^{b}= & \xi_{T+t}^{b}, \quad 0 \leq t \leq \delta, \\
Z_{T+t}^{b}= & P_{T+t}^{b}, \quad U_{T+t}^{b}=Q_{T+t}, \quad 0<t \leq \delta .
\end{aligned}\right.
$$

On the right-hand side of this equation, we still have to make precise if $Y_{t+\delta}$ is part of the solution before $\tau$ or after $\tau$, that is to separate the case $t+\delta<\tau$ and the case $t+\delta \geq \tau$. Furthermore, $U_{t}=\left[\left(Y_{t}^{a}(t)-Y_{t}^{b}\right) \mathbb{1}_{\{t \leq T\}}+\right.$ $\left.Q_{t} \mathbb{1}_{\{T<t \leq T+\delta\}}\right] \mathbb{1}_{\{t \leq \tau\}}$.

\subsection{Study of the Equation (2.1)}

Our aim is to write (2.1) as a family of ABSDEs in the filtration $\mathbb{F}$. For that purpose, we note that, on the set $\{t \geq \tau\}$, we have from (1.6)

$$
\mathbb{E}^{\mathcal{G}_{t}}\left[Y_{t+\delta}^{a}(\tau)\right]=\mathbb{E}^{\mathcal{F}_{t}}\left[Y_{t+\delta}^{a}(\theta)\right]_{\mid \theta=\tau} .
$$

The same equality holds for the part involving $f(t, \overrightarrow{\mathbf{y}})$ and $Z_{t+\delta}^{a}(\tau)$. Therefore, we study the family of ABSDE

$$
\left\{\begin{aligned}
-d Y_{t}^{a}(\theta)= & f^{a}\left(t, \theta, Y_{t}^{a}(\theta), \mathbb{E}^{\mathcal{F}_{t}}\left[Y_{t+\delta}^{a}(\theta)\right],\left\{\mathbb{E}^{\mathcal{F}_{t}}\left[Y_{t+s}^{a}(\theta)\right]\right\}_{0 \leq s \leq \delta}, Z_{t}^{a}(\theta), \mathbb{E}^{\mathcal{F}_{t}}\left[Z_{t+\delta}^{a}(\theta)\right],\right. \\
& \left.\left\{\mathbb{E}^{\mathcal{F}_{t}}\left[Z_{t+s}^{a}(\theta)\right]\right\}_{0 \leq s \leq \delta}, 0,0,0\right) d t-Z_{t}^{a}(\theta) d B_{t}, \quad 0 \leq t \leq T \\
Y_{T+t}^{a}(\theta)= & \xi_{T+t}^{a}(\theta), \quad 0 \leq t \leq \delta \\
Z_{T+t}^{a}(\theta)= & P_{T+t}^{a}(\theta), \quad 0<t \leq \delta
\end{aligned}\right.
$$

For any fixed $\theta \in[0, T]$, the map $F:=f^{a}(\theta)$ defined as $F(t, \overrightarrow{\mathbf{y}})=f^{a}(t, \theta, \overrightarrow{\mathbf{y}})$ inherits the Lipschitz conditions of Proposition 1.9 from the one of $f$. Due to the boundedness of $f(\cdot, \overrightarrow{\mathbf{0}})$, the map $F(\cdot, \overrightarrow{\mathbf{0}})$ is also bounded, and satisfies

$$
\sup _{0 \leq \theta \leq T} \mathbb{E}\left[\int_{0}^{T}\left|f^{a}(t, \theta, \overrightarrow{\mathbf{0}})\right|^{2} d t\right]<\infty,
$$

and the existence of a solution follows from Proposition 1.9.

Using Proposition 1.10, there exists a constant $K$ such that

$$
\begin{aligned}
\mathbb{E}^{\mathcal{F}_{t}}\left(\sup _{t \leq s \leq T}\left(Y_{s}^{a}(\theta)\right)^{2}+\int_{t}^{T}\left(Z_{s}^{a}(\theta)\right)^{2} d s\right) \leq & K \mathbb{E}^{\mathcal{F}_{t}}\left(\left(\xi_{T}^{a}(\theta)\right)^{2}+\int_{T}^{T+\delta}\left(\left(\xi_{s}^{a}(\theta)\right)^{2}+\left(P_{s}^{a}(\theta)\right)^{2}\right) d s\right. \\
& \left.+\int_{t}^{T}\left(f^{a}(s, \theta, \overrightarrow{\mathbf{0}})\right)^{2} d s\right) .
\end{aligned}
$$

\subsection{Study of the Equation (2.2)}

Our aim is to write (2.2) as an ABSDE in the filtration $\mathbb{F}$, that is to get rid of the quantities involving processes after time $\tau$ (as, e.g., $Y_{t+\delta}$ on $\{t+\delta>\tau\}$ ) and working only with conditional expectation w.r.t. $\mathbb{F}$. Obviously, for any $t \leq u \leq t+\delta$, we have

$$
\mathbb{E}^{\mathcal{G}_{t}}\left[Y_{u}\right]=\mathbb{E}^{\mathcal{G}_{t}}\left[Y_{u} \mathbb{1}_{\{u<\tau\}}\right]+\mathbb{E}^{\mathcal{G}_{t}}\left[Y_{u} \mathbb{1}_{\{u \geq \tau\}}\right]
$$

Furthermore, from (1.5), we have

$$
\mathbb{E}^{\mathcal{G}_{t}}\left[Y_{u} \mathbb{1}_{\{u<\tau\}}\right] \mathbb{1}_{\{t<\tau\}}=\mathbb{E}^{\mathcal{G}_{t}}\left[Y_{u}^{b} \mathbb{1}_{\{u<\tau\}}\right] \mathbb{1}_{\{t<\tau\}}=\frac{1}{G_{t}} \mathbb{E}^{\mathcal{F}_{t}}\left[Y_{u}^{b} G_{u}\right] \mathbb{1}_{\{t<\tau\}}
$$


and

$$
\begin{aligned}
\mathbb{E}^{\mathcal{G}_{t}}\left[Y_{u} \mathbb{1}_{\{u \geq \tau\}}\right] \mathbb{1}_{\{t<\tau\}} & =\mathbb{E}^{\mathcal{G}_{t}}\left[Y_{u}^{a}(\tau) \mathbb{1}_{\{u \geq \tau\}}\right] \mathbb{1}_{\{t<\tau\}} \\
& =\frac{1}{G_{t}} \mathbb{E}^{\mathcal{F}_{t}}\left[\int_{t}^{u} Y_{u}^{a}(\theta) \alpha_{u}(\theta) d \theta\right] \mathbb{1}_{\{t<\tau\}}=: J_{t}^{Y^{a}}(u) .
\end{aligned}
$$

The same equalities hold for the part involving $Z^{a}$. Then, we introduce, relying on the uniqueness of pre-default parts, the following BSDE which is a transformation of (2.2)

$$
\left\{\begin{aligned}
-d Y_{t}^{b} & =g\left(t, Y_{t}^{b}, Y_{t+\delta}^{b},\left\{Y_{t+s}^{b}\right\}_{0 \leq s \leq \delta}, Z_{t}^{b}, Z_{t+\delta}^{b},\left\{Z_{t+s}^{b}\right\}_{0 \leq s \leq \delta}\right) d t-Z_{t}^{b} d B_{t}, \quad 0 \leq t \leq T \\
Y_{T+t}^{b} & =\xi_{T+t}^{b}, \quad 0 \leq t \leq \delta \\
Z_{T+t}^{b} & =P_{T+t}^{b}, \quad 0<t \leq \delta .
\end{aligned}\right.
$$

Here, due to the equalities (2.5) and (2.6), $g$ is the map $\Omega \times[0, T] \times \mathbb{R} \times L^{2}\left(\mathcal{F}_{.+\delta}\right) \times \mathbb{D}(\cdot,[0, \delta]) \times \mathbb{R} \times L^{2}\left(\mathcal{F}_{\text {. }+\delta}\right) \times$ $\mathbb{D}(\cdot,[0, \delta]) \rightarrow \mathbb{R}$ defined, for $y$ and $z$ in $\mathbb{R}, \zeta$ and $\eta$ in $L^{2}\left(\mathcal{F}_{.+\delta}\right)$, and $\mathcal{Y}$ and $\mathcal{Z}$ in $\mathbb{D}(\cdot,[0, \delta])$, in terms of solution of the equation (2.3) by, for $\overrightarrow{\mathbf{y}}=(y, \zeta, \mathcal{Y}, z, \eta, \mathcal{Z})$

$$
g(t, \overrightarrow{\mathbf{y}})=f^{b}\left(t, \vec{I}_{t}^{1}, \vec{I}_{t}^{2}, \vec{I}_{t}^{3}\right)
$$

where, recalling that the quantities $J$ are defined in (2.6)

$$
\begin{aligned}
& \vec{I}_{t}^{1}=\left(y, \frac{1}{G_{t}} \mathbb{E}^{\mathcal{F}_{t}}\left[\zeta G_{t+\delta}\right]+J_{t}^{Y^{a}}(t+\delta),\left\{\frac{1}{G_{t}} \mathbb{E}^{\mathcal{F}_{t}}\left(\mathcal{Y}_{t}(s) G_{t+s}\right)+J_{t}^{Y^{a}}(t+s)\right\}_{0 \leq s \leq \delta}\right), \\
& \vec{I}_{t}^{2}=\left(z, \frac{1}{G_{t}} \mathbb{E}^{\mathcal{F}_{t}}\left[\eta G_{t+\delta}\right]+J_{t}^{Z^{a}}(t+\delta),\left\{\frac{1}{G_{t}} \mathbb{E}^{\mathcal{F}_{t}}\left(\mathcal{Z}_{t}(s) G_{t+s}\right)+J_{t}^{Z^{a}}(t+s)\right\}_{0 \leq s \leq \delta}\right), \\
& \vec{I}_{t}^{3}=\left(Y_{t}^{a}(t)-y, \frac{1}{G_{t}} \mathbb{E}^{\mathcal{F}_{t}}\left[\mathbb{1}_{\{t+\delta \leq T\}}\left(Y_{t+\delta}^{a}(t+\delta)-\zeta\right) G_{t+\delta}+\mathbb{1}_{\{t+\delta>T\}} Q_{t+\delta} G_{t+\delta}\right],\right. \\
& \left.\frac{1}{G_{t}}\left\{\mathbb{E}^{\mathcal{F}_{t}}\left[\left(Y_{t+s}^{a}(t+s)-\mathcal{Y}_{t}(s)\right) G_{t+s} \mathbb{1}_{\{t+s \leq T\}}+Q_{t+s} G_{t+s} \mathbb{1}_{\{t+s>T\}}\right]\right\}_{0 \leq s \leq \delta}\right) .
\end{aligned}
$$

It is straightforward that $g$ is $\mathbb{F}$-optional. We now show that $g$ satisfies Lipschitz conditions recalled in Proposition 1.9.

Since we have

$$
f^{b}(t, \overrightarrow{\mathbf{y}})=\frac{1}{G_{t}} \mathbb{E}^{\mathcal{F}_{t}}\left[f(t, \overrightarrow{\mathbf{y}}) \mathbb{1}_{\{t<\tau\}}\right]
$$

we obtain that, using the Lipschitz condition for $f$ and that $G$ is bounded, there exists a constant $K$ such that

$$
\begin{aligned}
\left|g(t, \overrightarrow{\mathbf{y}})-g\left(t, \overrightarrow{\mathbf{y}}^{\prime}\right)\right| \leq & \frac{K}{G_{t}}\left(\left(\left|y-y^{\prime}\right|+\left|z-z^{\prime}\right|\right) \mathbb{E}^{\mathcal{F}_{t}}\left(\mathbb{1}_{\{t<\tau\}}\right)\right. \\
& +\mathbb{E}^{\mathcal{F}_{t}}\left[\left(\left|\zeta-\zeta^{\prime}\right|+\left|\eta-\eta^{\prime}\right|\right) G_{t+\delta} \mathbb{1}_{\{t<\tau\}}\right] \\
& \left.+\mathbb{E}^{\mathcal{F}_{t}}\left[\left(\left|\mathcal{Y} G-\mathcal{Y}^{\prime} G\right|+\left|\mathcal{Z} G-\mathcal{Z}^{\prime} G\right|\right) \mathbb{1}_{\{t<\tau\}}\right]\right)
\end{aligned}
$$

Since, for $X \in \mathcal{F}_{s}$ and $s>t$, one has from (1.4)

$$
\mathbb{E}^{\mathcal{F}_{t}}\left[X \mathbb{1}_{\{t<\tau\}}\right]=\mathbb{E}^{\mathcal{F}_{t}}\left[X \mathbb{E}^{\mathcal{F}_{s}}\left(\mathbb{1}_{\{t<\tau\}}\right)\right]=\mathbb{E}^{\mathcal{F}_{t}}\left[X G_{t}\right]=G_{t} \mathbb{E}^{\mathcal{F}_{t}}[X]
$$


we deduce

$$
\begin{aligned}
\left|g(t, \overrightarrow{\mathbf{y}})-g\left(t, \overrightarrow{\mathbf{y}}^{\prime}\right)\right| \leq & K\left(\left|y-y^{\prime}\right|+\left|z-z^{\prime}\right|+\mathbb{E}^{\mathcal{F}_{t}}\left[\left(\left|\zeta-\zeta^{\prime}\right|+\left|\eta-\eta^{\prime}\right|\right) G_{t+\delta}\right]\right. \\
& \left.+\mathbb{E}^{\mathcal{F}_{t}}\left[\left|\mathcal{Y} G-\mathcal{Y}^{\prime} G\right|+\left|\mathcal{Z} G-\mathcal{Z}^{\prime} G\right|\right]\right) .
\end{aligned}
$$

Noting $G$ is upper bounded by 1 , the Lipschitz property of Proposition 1.9 for $g$ holds.

We now check the integrability condition on $|g(t, \overrightarrow{\mathbf{0}})|^{2}$. We notice, using notation $(2.6)$, we have

$$
\begin{aligned}
g(t, \overrightarrow{\mathbf{0}})= & f^{b}\left(t, 0, J_{t}^{Y^{a}}(t+\delta),\left\{J_{t}^{Y^{a}}(t+s)\right\}_{0 \leq s \leq \delta}, 0, J_{t}^{Z^{a}}(t+\delta),\left\{J_{t}^{Z^{a}}(t+s)\right\}_{0 \leq s \leq \delta}, Y_{t}^{a}(t),\right. \\
& \frac{1}{G_{t}} \mathbb{E}^{\mathcal{F}_{t}}\left[Y_{t+\delta}^{a}(t+\delta) G_{t+\delta} \mathbb{1}_{\{t+\delta \leq T\}}+Q_{t+\delta} G_{t+\delta} \mathbb{1}_{\{t+\delta>T\}}\right] \\
& \left.\left\{\frac{1}{G_{t}} \mathbb{E}^{\mathcal{F}_{t}}\left[Y_{t+s}^{a}(t+s) G_{t+s} \mathbb{1}_{\{t+s \leq T\}}+Q_{t+s} G_{t+s} \mathbb{1}_{\{t+s>T\}}\right]\right\}_{0 \leq s \leq \delta}\right)
\end{aligned}
$$

From Lipschitz property of $f$, since $f(t, \overrightarrow{\mathbf{0}})$ is bounded and $G_{t}=\mathbb{E}^{\mathcal{F}_{t}}\left(\mathbb{1}_{\{t<\tau\}}\right)$, we have

$$
f^{b}(t, \overrightarrow{\mathbf{y}}) \leq \frac{1}{G_{t}}\left(\mathbb{E}^{\mathcal{F}_{t}}\left[(f(t, \overrightarrow{\mathbf{0}})+C|\overrightarrow{\mathbf{y}}|) \mathbb{1}_{\{t<\tau\}}\right]\right) \leq C_{1}+C|\overrightarrow{\mathbf{y}}|
$$

Using again that the square of a sum is bounded (up to a constant) by the sum of the squares, and using again the fact that $G$ is lower bounded, the integrability condition of $|g(t, \overrightarrow{\mathbf{0}})|^{2}$ will follow from the boundedness of the quantities

$$
\mathbb{E}\left(\int_{0}^{T}\left(J_{t}^{Y}(t+\delta)\right)^{2} d t\right), \quad \mathbb{E}\left(\int_{0}^{T} \int_{0}^{\delta}\left(J_{t}^{Y}(t+s)\right)^{2} d s d t\right)
$$

and similar expressions with $J^{Z}$, as well as

$$
\left\{\begin{array}{l}
\mathbb{E}\left(\int_{0}^{T}\left(\mathbb{E}^{\mathcal{F}_{t}}\left(Y_{t+\delta}^{a}(t+\delta)\right)\right)^{2} d t\right) \\
\mathbb{E}\left(\int_{0}^{T}\left(Y_{t}^{a}(t)\right)^{2} d t\right) \\
\mathbb{E}\left(\int_{T}^{T+\delta}\left(\mathbb{E}^{\mathcal{F}_{t}}\left(Q_{t}\right)\right)^{2} d t\right) \\
\mathbb{E}\left(\int_{0}^{T}\left[\int_{0}^{\delta}\left(Y_{t+s}^{a}(t+s)^{2} \mathbb{1}_{t+s \leq T}+Q_{t+s}^{2} \mathbb{1}_{t+s>T}\right) d s\right] d t\right) .
\end{array}\right.
$$

The quantities in (2.8) are bounded since $\alpha$ is bounded and

$$
\int_{0}^{T+\delta} d \theta \int_{0}^{T} \mathbb{1}_{\{t<\theta<t+\delta\}} \mathbb{E}\left(\left(Y_{t+\delta}^{a}(\theta)\right)^{2}\right) d t
$$

is bounded since

$$
\begin{aligned}
\sup _{0 \leq \theta \leq T} \mathbb{E}\left(\left(\sup _{0 \leq s \leq T} Y_{s}^{a}(\theta)\right)^{2}\right) \leq & K \sup _{0 \leq \theta \leq T} \mathbb{E}\left(\left(\xi_{T}^{a}(\theta)\right)^{2}+\int_{T}^{T+\delta}\left(\left(\xi_{s}^{a}(\theta)\right)^{2}+\left(P_{s}^{a}(\theta)\right)^{2}\right) d s\right. \\
& \left.+\int_{0}^{T}\left(f^{a}(s, \theta, \overrightarrow{\mathbf{0}})\right)^{2} d s\right)
\end{aligned}
$$

and the assumed boundedness of $P$ and $\xi$. The other quantities are studied using the same methodology and that $Q \in L^{2}[T, T+\delta]$. 
The existence of a unique solution $\left(Y^{b}, Z^{b}\right)$ of the ABSDE (2.7) follows from Proposition 1.11. Moreover we have

$$
\begin{aligned}
\mathbb{E}^{\mathcal{F}_{t}}\left(\sup _{t \leq s \leq T}\left(Y_{s}^{b}\right)^{2}+\int_{t}^{T}\left(Z_{s}^{b}\right)^{2} d s\right) \leq & K \mathbb{E}^{\mathcal{F}_{t}}\left(\left|\xi_{T}^{b}\right|^{2}+\int_{T}^{T+\delta}\left(\left(\xi_{s}^{b}\right)^{2}+\left(P_{s}^{b}\right)^{2}\right) d u\right. \\
& \left.+\int_{t}^{T}\left(f^{b}(s, \overrightarrow{\mathbf{0}})\right)^{2} d s\right)
\end{aligned}
$$

\subsection{Integrability of the solutions}

In this part we consider the integrability of the solutions $(Y, Z, U)$ where

$$
\begin{aligned}
Y_{t} & =Y_{t}^{b} \mathbb{1}_{\{t<\tau\}}+Y_{t}^{a}(\tau) \mathbb{1}_{\{t \geq \tau\}}, \\
Z_{t} & =Z_{t}^{b} \mathbb{1}_{\{t \leq \tau\}}+Z_{t}^{a}(\tau) \mathbb{1}_{\{t>\tau\}}, \\
U_{t} & =\left(Y_{t}^{a}(t)-Y_{t}^{b}\right) \mathbb{1}_{\{t \leq \tau\}} .
\end{aligned}
$$

From Subsections 2.1 and 2.2 we know $(Y, Z, U)$ satisfy the ABSDE (0.1).

Proposition 2.2. The process $U$ belongs to $L_{\tau}^{2}$.

Proof. We have

$$
\begin{aligned}
\mathbb{E}\left[\int_{0}^{(T+\delta) \wedge \tau} U_{s}^{2} d s\right] & =\mathbb{E}\left[\int_{0}^{T \wedge \tau}\left(Y_{s}^{a}(s)-Y_{s}^{b}\right)^{2} d s\right]+\left[\int_{T \wedge \tau}^{(T+\delta) \wedge \tau} Q_{s}^{2} d s\right] \\
& \leq 2 \mathbb{E}\left[\int_{0}^{T}\left(Y_{s}^{a}(s)\right)^{2} d s\right]+2 \mathbb{E}\left[\int_{0}^{T}\left(Y_{s}^{b}\right)^{2} d s\right]+\left[\int_{T}^{T+\delta} Q_{s}^{2} d s\right] \\
& \leq 2 \int_{0}^{T} \mathbb{E}\left[\left(Y_{s}^{a}(s)\right)^{2}\right] d s+2 T \mathbb{E}\left[\sup _{0 \leq t \leq T}\left(Y_{t}^{b}\right)^{2}\right]+\mathbb{E}\left[\int_{T}^{T+\delta} Q_{s}^{2} d s\right]
\end{aligned}
$$

and the quantities on the right-hand side are finite.

Proposition 2.3. There exists a strictly positive constant $K$ such that the solution $(Y, Z, U)$ of the ABSDE (0.1) satisfies

$$
\begin{aligned}
& \mathbb{E}^{\mathcal{G}_{t}}\left[\sup _{t \leq s \leq T} Y_{s}^{2}+\int_{t}^{T} Z_{s}^{2} d s\right] \\
\leq & K \mathbb{E}^{\mathcal{F}_{t}}\left[\left(\xi_{T}^{b}\right)^{2}+\int_{T}^{T+\delta}\left(\left(\xi_{s}^{b}\right)^{2}+\left(P_{s}^{b}\right)^{2}\right) d s+\int_{t}^{T}\left(f^{b}(s, \overrightarrow{0})\right)^{2} d s\right] \\
+ & \frac{K}{\alpha_{t}(\tau)} \mathbb{E}^{\mathcal{F}_{t}}\left[\left(\xi_{T}^{a}(\theta)\right)^{2}+\int_{T}^{T+\delta}\left(\left(\xi_{s}^{a}(\theta)\right)^{2}+\left(P_{s}^{a}(\theta)\right)^{2}\right) d s+\int_{t}^{T}\left(f^{a}(s, \theta, \overrightarrow{0})\right)^{2} d s\right]_{\theta=\tau} \mathbb{1}_{\{\tau<t\}} \\
+ & K \mathbb{1}_{\{t \leq \tau\}} \mathbb{E}^{\mathcal{F}_{t}}\left[\int_{t}^{T}\left\{\left(\xi_{T}^{a}(\theta)\right)^{2}+\int_{T}^{T+\delta}\left(\left(\xi_{s}^{a}(\theta)\right)^{2}+\left(P_{s}^{a}(\theta)\right)^{2}\right) d s+\int_{t}^{T}\left(f^{a}(s, \theta, \overrightarrow{0})\right)^{2} d s\right\} d \theta\right]
\end{aligned}
$$

for any $t \in[0, T]$. 
Proof. In the proof, the constant $K$ can vary from line to line. We remark ${ }^{1}$

$$
\begin{aligned}
& \mathbb{E}^{\mathcal{G}_{t}}\left[\sup _{t \leq s \leq T} Y_{s}^{2}+\int_{t}^{T} Z_{s}^{2} d s\right] \\
= & \mathbb{E}^{\mathcal{G}_{t}}\left[\sup _{t \leq s \leq T} Y_{s}^{2}+\int_{t}^{T \wedge \tau}\left(Z_{s}^{b}\right)^{2} d s+\int_{T \wedge \tau}^{T}\left(Z_{s}^{a}(\tau)\right)^{2} d s\right] \\
\leq & \mathbb{E}^{\mathcal{G}_{t}}\left[\sup _{t \leq s \leq T} Y_{s}^{2}+\int_{t}^{T}\left(Z_{s}^{b}\right)^{2} d s+\int_{T \wedge \tau}^{T}\left(Z_{s}^{a}(\tau)\right)^{2} d s\right] .
\end{aligned}
$$

On the set $\{\tau<t\}$, since $\lambda$ is bounded (Hypothesis 1.3), we use that

$$
\begin{aligned}
\mathbb{E}^{\mathcal{G}_{t}}\left[\sup _{t \leq s \leq T} Y_{s}^{2}\right] & =\mathbb{E}^{\mathcal{G}_{t}}\left[\sup _{t \leq s \leq T}\left(Y_{s}^{a}(\tau)\right)^{2}\right]=\frac{1}{G_{t}} \mathbb{E}^{\mathcal{F}_{t}}\left[\sup _{t \leq s \leq T}\left(Y_{s}^{a}(\theta)\right)^{2} \alpha_{T}(\theta)\right] \\
& \leq k e^{k t} \mathbb{E}^{\mathcal{F}_{t}}\left[\sup _{t \leq s \leq T}\left(Y_{s}^{a}(\theta)\right)^{2}\right] \leq K \mathbb{E}^{\mathcal{F}_{t}}\left[\sup _{t \leq s \leq T}\left(Y_{s}^{a}(\theta)\right)^{2}\right] .
\end{aligned}
$$

On the set $\{t \leq \tau\}$, we remark

$$
\mathbb{E}^{\mathcal{G}_{t}}\left[\sup _{t \leq s \leq T} Y_{s}^{2}\right] \leq \mathbb{E}^{\mathcal{F}_{t}}\left[\sup _{t \leq s \leq T}\left(Y_{s}^{b}\right)^{2}\right]+\mathbb{E}^{\mathcal{G}_{t}}\left[\sup _{T \wedge \tau \leq s \leq T}\left(Y_{s}^{a}(\tau)\right)^{2}\right] .
$$

From $\mathbb{E}^{\mathcal{G}_{t}}\left[\sup _{T \wedge \tau \leq s \leq T}\left(Y_{s}^{a}(\tau)\right)^{2}\right]=\frac{1}{\alpha_{t}(\tau)} \mathbb{E}^{\mathcal{F}_{t}}\left[\sup _{T \wedge \theta \leq s \leq T}\left(Y_{s}^{a}(\theta)\right)^{2} \alpha_{T}(\theta)\right]_{\theta=\tau}$ and that $\alpha$ is bounded, we have

$$
\mathbb{E}^{\mathcal{G}_{t}}\left[\sup _{T \wedge \tau \leq s \leq T}\left(Y_{s}^{a}(\tau)\right)^{2}\right] \leq \frac{K}{\alpha_{t}(\tau)} \mathbb{E}^{\mathcal{F}_{t}}\left[\sup _{T \wedge \theta \leq s \leq T}\left(Y_{s}^{a}(\theta)\right)^{2}\right]_{\theta=\tau}
$$

We proceed in the same way for the part $\int_{T \wedge \tau}^{T}\left(Z_{s}^{a}(\tau)\right)^{2} d s$.

Using (2.4)-(2.9) we can conclude.

\subsection{Uniqueness of the solution}

In this part we are concerned with the uniqueness of the solution of ABSDE (0.1). Suppose this ABSDE has two solutions $(Y, Z, U)$ and $(\bar{Y}, \bar{Z}, \bar{U})$. Each process admits a unique decomposition under the form $\left(Y^{b}, Z^{b}, U^{b}\right)-\left(Y^{a}(\tau), Z^{a}(\tau)\right)$ and $\left(\bar{Y}^{b}, \bar{Z}^{b}, \bar{U}^{b}\right)-\left(\bar{Y}^{a}(\tau), \bar{Z}^{a}(\tau)\right)$. Moreover we know $\left(Y^{b}, Z^{b}\right)$ and $\left(\bar{Y}^{b}, \bar{Z}^{b}\right)$ are solution of ABSDE (2.2), thus by uniqueness of the solution of ABSDE (2.2) from Proposition 1.11 we get that $Y^{b}=\bar{Y}^{b}$ and $Z^{b}=\bar{Z}^{b}$. We have with the same arguments $Y^{a}(\tau)=\bar{Y}^{a}(\tau)$ and $Z^{a}(\tau)=\bar{Z}^{a}(\tau)$. Moreover we have $U_{t}=\left(Y_{t}^{a}(t)-Y_{t}^{b}\right) \mathbb{1}_{\{t \leq \tau\}}$, thus $U=\bar{U}$. Finally we get the uniqueness of the solution of ABSDE (0.1).

\section{ABSDE With JUMP OF TYPE (0.2)}

We assume that Hypotheses 1.1, 1.2 and 1.3 hold. We define, for any $t \in[0, T], \mathbf{A}_{t}=\mathbb{R} \times L^{2}\left(\mathcal{F}_{t+\delta}\right) \times$ $\mathbb{D}(t,[0, \delta]) \times \mathbb{R} \times L^{2}\left(\mathcal{F}_{t+\delta}\right) \times \mathbb{D}(t,[0, \delta]) \times \mathbb{R} \times L^{2}\left(\mathcal{F}_{t+\delta}\right) \times \mathbb{D}(t,[0, \delta])$. We consider in this section an ABSDE of the following form: find a triple $(Y, Z, U) \in \mathcal{S}_{\mathbb{G}}^{2}[0, T+\delta] \times L_{\mathbb{G}}^{2}[0, T+\delta] \times L_{\tau}^{2}$ satisfying

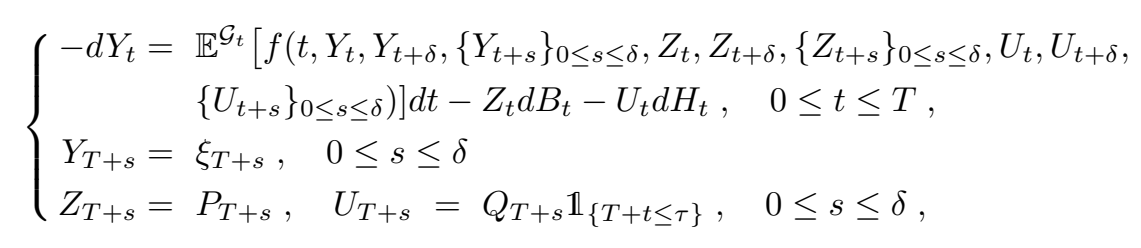

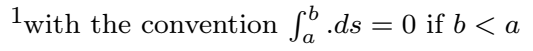


with the following hypotheses

Hypotheses 3.1. Suppose that

a) The terminal conditions satisfy $\xi \in \mathcal{S}_{\mathbb{G}}^{2}[T, T+\delta], P \in L_{\mathbb{G}}^{2}[T, T+\delta]$ and $Q \in L_{\mathbb{F}}^{2}[T, T+\delta]$, and $\sup _{0 \leq \theta \leq T} \xi^{a}(\theta) \in$ $\mathcal{S}_{\mathbb{F}}^{2}[T, T+\delta]$ and $\sup _{0 \leq \theta \leq T} P^{a}(\theta) \in L_{\mathbb{F}}^{2}[T, T+\delta]$.

b) The driver $f: \Omega \times[0, T] \times \mathbf{A} \rightarrow \mathbb{R}$ is Lipschitz, that means there exists a constant $C$ such that for any $t \in[0, T]$, for any $\overrightarrow{\mathbf{y}}$ and $\overrightarrow{\mathbf{y}}^{\prime}$ in $\mathbf{A}_{t}$, one has

$$
\left|f(t, \overrightarrow{\mathbf{y}})-f\left(t, \overrightarrow{\mathbf{y}}^{\prime}\right)\right| \leq C\left|\overrightarrow{\mathbf{y}}-\overrightarrow{\mathbf{y}}^{\prime}\right| .
$$

c) There exists a constant $C^{\prime}$ such that $|f(s, \overrightarrow{\mathbf{0}})| \leq C^{\prime}$.

Proceeding as before, we consider, on the set $\{\tau \leq t\}$, the ABSDE

$$
\left\{\begin{aligned}
-d Y_{t}^{a}(\tau)= & \mathbb{E}^{\mathcal{G}_{t}}\left[f ^ { a } \left(t, \tau, Y_{t}^{a}(\tau), Y_{t+\delta}^{a}(\tau),\left\{Y_{t+s}^{a}(\tau)\right\}_{0 \leq s \leq \delta}, Z_{t}^{a}(\tau), Z_{t+\delta}^{a}(\tau)\right.\right. \\
& \left.\left.\left\{Z_{t+s}^{a}(\tau)\right\}_{0 \leq s \leq \delta}, 0,0,0\right)\right] d t-Z_{t}^{a}(\tau) d B_{t}, \quad \tau \leq t \leq T \\
Y_{T+s}^{a}(\tau)= & \xi_{T+s}^{a}(\tau), \quad 0 \leq s \leq \delta \\
Z_{T+s}^{a}(\tau)= & P_{T+s}^{a}(\tau), \quad 0<s \leq \delta
\end{aligned}\right.
$$

whereas, due to the uniqueness of pre-default parts we consider the ABSDE

$$
\left\{\begin{aligned}
-d Y_{t}^{b}= & \mathbb{E}^{\mathcal{G}_{t}}\left[f^{b}\left(t, Y_{t}^{b}, Y_{t+\delta},\left\{Y_{t+s}\right\}_{0 \leq s \leq \delta}, Z_{t}^{b}, Z_{t+\delta},\left\{Z_{t+s}\right\}_{0 \leq s \leq \delta}, U_{t}^{b}, U_{t+\delta},\left\{U_{t+s}\right\}_{0 \leq s \leq \delta}\right)\right] d t \\
& -Z_{t}^{b} d B_{t}, \quad 0 \leq t \leq T, \\
Y_{T+s}^{b}= & \xi_{T+s}^{b}, \quad 0 \leq s \leq \delta \\
Z_{T+s}^{b}= & P_{T+s}, \quad U_{T+s}^{b}=Q_{T+s}, \quad 0<s \leq \delta .
\end{aligned}\right.
$$

\subsection{Study of the Equation (3.2)}

Using the same arguments as in Subsection 2.1 we study the family of ABSDEs

$$
\left\{\begin{aligned}
-d Y_{t}^{a}(\theta)= & \mathbb{E}^{\mathcal{F}_{t}}\left[f ^ { a } \left(t, \theta, Y_{t}^{a}(\theta), Y_{t+\delta}^{a}(\theta),\left\{Y_{t+s}^{a}(\theta)\right\}_{0 \leq s \leq \delta}, Z_{t}^{a}(\theta), Z_{t+\delta}^{a}(\theta),\right.\right. \\
& \left.\left.\left\{Z_{t+s}^{a}(\theta)\right\}_{0 \leq s \leq \delta}, 0,0,0\right)\right] d t-Z_{t}^{a}(\theta) d B_{t}, \quad \theta \leq t \leq T \\
Y_{T+t}^{a}(\theta)= & \xi_{T+t}^{a}(\theta), \quad 0 \leq t \leq \delta \\
Z_{T+t}^{a}(\theta)= & P_{T+t}^{a}(\theta), \quad 0<t \leq \delta
\end{aligned}\right.
$$

This ABSDE can be written under the following form

$$
\left\{\begin{aligned}
-d Y_{t}^{a}(\theta)= & g\left(t, \theta, Y_{t}^{a}(\theta), Y_{t+\delta}^{a}(\theta),\left\{Y_{t+s}^{a}(\theta)\right\}_{0 \leq s \leq \delta}, Z_{t}^{a}(\theta), Z_{t+\delta}^{a}(\theta),\left\{Z_{t+s}^{a}(\theta)\right\}_{0 \leq s \leq \delta}\right) d t \\
& -Z_{t}^{a}(\theta) d B_{t}, \quad \theta \leq t \leq T \\
Y_{T+t}^{a}(\theta)= & \xi_{T+t}^{a}(\theta), \quad 0 \leq t \leq \delta \\
Z_{T+t}^{a}(\theta)= & P_{T+t}^{a}(\theta), \quad 0<t \leq \delta
\end{aligned}\right.
$$

which is on the form of Proposition 1.11, where $g$ is given by

$$
g(t, \theta, y, \hat{y}, \mathcal{Y}, z, \hat{z}, \mathcal{Z})=\mathbb{E}^{\mathcal{F}_{t}}\left[f^{a}(t, \theta, y, \hat{y}, \mathcal{Y}, z, \hat{z}, \mathcal{Z}, 0,0,0)\right]
$$


The Lipschitz condition on $g$ follows from the hypothesis on $f$. The square integrability of $g(t, \overrightarrow{\mathbf{0}})=$ $\mathbb{E}^{\mathcal{F}_{t}}\left[f^{a}(t, \theta, \overrightarrow{\mathbf{0}})\right]$ follows as in Subsection 2.1 from the boundedness hypothesis of $f(t, \overrightarrow{\mathbf{0}})$. Thus from Proposition 1.11 we get the existence of a unique solution to this ABSDE satisfying

$$
\begin{aligned}
\mathbb{E}^{\mathcal{F}_{t}}\left(\sup _{t \leq s \leq T}\left(Y_{s}^{a}(\theta)\right)^{2}+\int_{t}^{T}\left(Z_{s}^{a}(\theta)\right)^{2} d s\right) \leq & C \mathbb{E}^{\mathcal{F}_{t}}\left(\left(\xi_{T}^{a}(\theta)\right)^{2}+\int_{T}^{T+\delta}\left(\left(\xi_{s}^{a}(\theta)\right)^{2}+\left(P_{s}^{a}(\theta)\right)^{2}\right) d s\right. \\
& \left.+\int_{t}^{T}(g(s, \theta, \overrightarrow{\mathbf{0}}))^{2} d s\right) .
\end{aligned}
$$

\subsection{Study of the Equation (3.3)}

Using the same arguments as in Subsection 2.2, we are lead to consider

$$
\left\{\begin{aligned}
-d Y_{t}^{b} & =g\left(t, Y_{t}^{b}, Y_{t+\delta}^{b},\left\{Y_{t+s}^{b}\right\}_{0 \leq s \leq \delta}, Z_{t}^{b}, Z_{t+\delta}^{b},\left\{Z_{t+s}^{b}\right\}_{0 \leq s \leq \delta}\right) d t-Z_{t}^{b} d B_{t}, \quad 0 \leq t \leq T \\
Y_{T+s}^{b} & =\xi_{T+s}^{b}, \quad 0 \leq s \leq \delta \\
Z_{T+s}^{b} & =P_{T+s}^{b}, \quad 0<s \leq \delta
\end{aligned}\right.
$$

where

$$
\begin{aligned}
g(t, y, \zeta, \mathcal{Y}, z, \eta, \mathcal{Z})= & \frac{1}{G_{t}} \int_{t}^{t+\delta} \mathbb{E}^{\mathcal{F}_{t}}\left[f^{b}\left(t, \vec{K}_{t}^{1}, \vec{K}_{t}^{2}, \vec{K}_{t}^{3}\right) \alpha_{t+\delta}(\theta)\right] d \theta \\
& +\frac{1}{G_{t}} \mathbb{E}^{\mathcal{F}_{t}}\left[f^{b}\left(t, y, \zeta, \mathcal{Y}, z, \eta, \mathcal{Z}, \vec{K}_{t}^{4}\right)\right]
\end{aligned}
$$

with

$$
\begin{aligned}
\vec{K}_{t}^{1}= & \left(y, Y_{t+\delta}^{a}(\theta),\left\{\mathcal{Y}_{s} \mathbb{1}_{t+s<\theta}+Y_{t+s}^{a}(\theta) \mathbb{1}_{t+s \geq \theta}\right\}_{0 \leq s \leq \delta}\right) \\
\vec{K}_{t}^{2}= & \left(z, Z_{t+\delta}^{a}(\theta),\left\{\mathcal{Z}_{s} \mathbb{1}_{t+s \leq \theta}+Z_{t+s}^{a}(\theta) \mathbb{1}_{t+s>\theta}\right\}_{0 \leq s \leq \delta}\right) \\
\vec{K}_{t}^{3}= & \left(Y_{t}^{a}(t)-y, 0,\left\{\left(\left(Y_{t+s}^{a}(t+s)-\mathcal{Y}_{s}\right) \mathbb{1}_{t+s<T}+Q_{t+s} \mathbb{1}_{t+s \geq T}\right) \mathbb{1}_{t+s \leq \theta}\right\}_{0 \leq s \leq \delta}\right) \\
\vec{K}_{t}^{4}= & \left(Y_{t}^{a}(t)-y,\left(\left(Y_{t+\delta}^{a}(t+\delta)-\zeta\right) \mathbb{1}_{\{t+\delta \leq T\}}+Q_{t+\delta} \mathbb{1}_{\{t+\delta>T\}}\right) G_{t+\delta},\right. \\
& \left.\left\{\left(\left(Y_{t+s}^{a}(t+s)-\mathcal{Y}_{s}\right) \mathbb{1}_{\{t+s \leq T\}}+Q_{t+s} \mathbb{1}_{\{t+s>T\}}\right) G_{t+s}\right\}_{0 \leq s \leq \delta}\right) .
\end{aligned}
$$

We show that the hypotheses of Proposition 1.11 are satisfied. First, we show that the driver is Lipschitz. Using that $f$ is Lipschitz we get

$$
\left|f^{b}(t, \overrightarrow{\mathbf{y}})-f^{b}\left(t, \overrightarrow{\mathbf{y}}^{\prime}\right)\right| \leq \frac{1}{G_{t}}\left|\mathbb{E}^{\mathcal{F}_{t}}\left[\left|f(t, \overrightarrow{\mathbf{y}})-f\left(t, \overrightarrow{\mathbf{y}}^{\prime}\right)\right| \mathbb{1}_{\{t<\tau\}}\right]\right| \leq C\left|\overrightarrow{\mathbf{y}}-\overrightarrow{\mathbf{y}}^{\prime}\right| .
$$

It follows that, setting

$$
\begin{gathered}
\overrightarrow{\mathbf{Y}}=\left(y, Y_{t+\delta}^{a}(\theta),\left\{\mathcal{Y}_{s} \mathbb{1}_{t+s<\theta}+Y_{t+s}^{a}(\theta) \mathbb{1}_{t+s \geq \theta}\right\}_{0 \leq s \leq \delta}, z, Z_{t+\delta}^{a}(\theta),\left\{\mathcal{Z}_{s} \mathbb{1}_{t+s \leq \theta}+Z_{t+s}^{a}(\theta) \mathbb{1}_{t+s>\theta}\right\}_{0 \leq s \leq \delta}\right. \\
\left.Y_{t}^{a}(t)-y, 0,\left\{\left(\left(Y_{t+s}^{a}(t+s)-\mathcal{Y}_{s}\right) \mathbb{1}_{t+s<T}+Q_{t+s} \mathbb{1}_{t+s \geq T}\right) \mathbb{1}_{t+s \leq \theta}\right\}_{0 \leq s \leq \delta}\right)
\end{gathered}
$$


there exists a constant $C$ such that

$$
\begin{aligned}
\mid \mathbb{E}^{\mathcal{F}_{t}} & {\left[f^{b}(t, \overrightarrow{\mathbf{Y}}) \alpha_{t+\delta}(\theta)\right]-\mathbb{E}^{\mathcal{F}_{t}}\left[f^{b}\left(t, \overrightarrow{\mathbf{Y}}^{\prime}\right) \alpha_{t+\delta}(\theta)\right] \mid } \\
& \leq C\left(\left(\left|y-y^{\prime}\right|+\left|z-z^{\prime}\right|\right) \mathbb{E}^{\mathcal{F}_{t}}\left(\alpha_{t+\delta}(\theta)\right)+\left|\mathcal{Y} \alpha(\theta)-\mathcal{Y}^{\prime} \alpha(\theta)\right|+\left|\mathcal{Z} \alpha(\theta)-\mathcal{Z}^{\prime} \alpha(\theta)\right|\right) \\
& \leq C\left(\left|y-y^{\prime}\right|+\left|z-z^{\prime}\right|+\left|\mathcal{Y}-\mathcal{Y}^{\prime}\right|+\left|\mathcal{Z}-\mathcal{Z}^{\prime}\right|\right) \alpha_{t}(\theta),
\end{aligned}
$$

where we use that $\alpha(\theta)$ is a martingale. Hence, using that $\int_{0}^{\infty} \alpha_{t}(\theta) d \theta=1$, we get

$$
\int_{t}^{t+\delta} \mathbb{E}^{\mathcal{F}_{t}}\left[\left|f^{b}(t, \overrightarrow{\mathbf{Y}})-f^{b}\left(t, \overrightarrow{\mathbf{Y}}^{\prime}\right)\right| \alpha_{t+\delta}(\theta)\right] d \theta \quad \leq \quad C\left(\left|y-y^{\prime}\right|+\left|z-z^{\prime}\right|+\left|\mathcal{Y}-\mathcal{Y}^{\prime}\right|+\left|\mathcal{Z}-\mathcal{Z}^{\prime}\right|\right)
$$

In the other hand, using the Lipschitz property of $f^{b}$, that $G$ is upper bounded and denoting

$$
\varphi(t, \overrightarrow{\mathbf{y}}):=\mathbb{E}^{\mathcal{F}_{t}}\left[f^{b}(t, \overrightarrow{\mathbf{y}})\right]
$$

for $\overrightarrow{\mathbf{y}}=\left(y, \zeta, \mathcal{Y}, z, \eta, \mathcal{Z}, Y_{t}^{a}(t)-y,\left(\left(Y_{t+\delta}^{a}(t+\delta)-\zeta\right) \mathbb{1}_{\{t+\delta \leq T\}}+Q_{t+\delta} \mathbb{1}_{\{t+\delta>T\}}\right) G_{t+\delta},\left\{\left(\left(Y_{t+s}^{a}(t+s)-\mathcal{Y}_{s}\right) \mathbb{1}_{\{t+s \leq T\}}+\right.\right.\right.$ $\left.\left.\left.Q_{t+s} \mathbb{1}_{\{t+s>T\}}\right) G_{t+s}\right\}_{0 \leq s \leq \delta}\right)$ there exists a constant $K$ such that one has

$$
\left|\varphi(t, \overrightarrow{\mathbf{y}})-\varphi\left(t, \overrightarrow{\mathbf{y}}^{\prime}\right)\right| \leq K\left|\overrightarrow{\mathbf{y}}-\overrightarrow{\mathbf{y}}^{\prime}\right|
$$

It follows (using one more time that $G$ is lower bounded) that there exists a constant $K$ such that

$$
\left|g(t, \overrightarrow{\mathbf{y}})-g\left(t, \overrightarrow{\mathbf{y}}^{\prime}\right)\right| \leq K\left|\overrightarrow{\mathbf{y}}-\overrightarrow{\mathbf{y}}^{\prime}\right|
$$

and the Lipschitz property holds.

There exists an unique solution of (3.3).

\subsection{Integrability}

The integrability condition of

$$
\begin{aligned}
g(t, \overrightarrow{\mathbf{0}})= & \frac{1}{G_{t}} \int_{t}^{t+\delta} \mathbb{E}^{\mathcal{F}_{t}}\left[f ^ { b } \left(t, 0, Y_{t+\delta}^{a}(\theta),\left\{Y_{t+s}^{a}(\theta) \mathbb{1}_{t+s \geq \theta}\right\}_{0 \leq s \leq \delta}\right.\right. \\
& 0, Z_{t+\delta}^{a}(\theta),\left\{Z_{t+s}^{a}(\theta) \mathbb{1}_{t+s \geq \theta}\right\}_{0 \leq s \leq \delta} \\
& \left.\left.Y_{t}^{a}(t), 0,\left\{\left(Y_{t+s}^{a}(t+s) \mathbb{1}_{\{t+s \leq T\}}+Q_{t+s} \mathbb{1}_{\{t+s>T\}}\right) \mathbb{1}_{t+s \leq \theta}\right\}_{0 \leq s \leq \delta}\right) \alpha_{t+\delta}(\theta)\right] d \theta \\
+ & \frac{1}{G_{t}} \mathbb{E}^{\mathcal{F}_{t}}\left[f^{b}\left(t, 0,0,0,0, Y_{t}^{a}(t), Y_{t+\delta}^{a}(t+\delta) G_{t+\delta} \mathbb{1}_{\{t+\delta<T\}}+Q_{t+\delta} G_{t+\delta} \mathbb{1}_{\{t+\delta \geq T\}}\right)\right]
\end{aligned}
$$

follows with the same arguments as in Section 2.2.

We also consider the integrability of the solutions $(Y, Z, U)$ for $\operatorname{ABSDE}(3.1)$, where

$$
\begin{aligned}
Y_{t} & =Y_{t}^{b} \mathbb{1}_{\{t<\tau\}}+Y_{t}^{a}(\tau) \mathbb{1}_{\{t \geq \tau\}}, \\
Z_{t} & =Z_{t}^{b} \mathbb{1}_{\{t \leq \tau\}}+Z_{t}^{a}(\tau) \mathbb{1}_{\{t>\tau\}}, \\
U_{t} & =\left(Y_{t}^{a}(t)-Y_{t}^{b}\right) \mathbb{1}_{\{t \leq \tau\}} .
\end{aligned}
$$

One can apply the same methodology than the one in the previous section, since Proposition 1.10 is valid in the case of ABSDE (3.1) and we obtain similar results. 


\section{Particular cases}

\subsection{Moving average term}

We consider the case

$$
\left\{\begin{array}{l}
-d Y_{t}=\mathbb{E}^{\mathcal{G}_{t}}\left[f\left(t, Y_{t}, \int_{t}^{t+\delta} a_{s} Y_{s} \eta(d s), Z_{t}, U_{t}\right)\right] d t-Z_{t} d B_{t}-U_{t} d H_{t}, \quad 0 \leq t \leq T, \\
Y_{T+t}=\xi_{T+t}, \quad 0 \leq t \leq \delta, \\
Z_{T+t}=P_{T+t}, \\
U_{T+t}=Q_{T+t} \mathbb{1}_{\{T+t \leq \tau\}} \quad 0<t \leq \delta,
\end{array}\right.
$$

where $a$ is a bounded $\mathbb{F}$-adapted process and $\eta$ is a measure of the form $\eta(d s)=\ell(s) d s+\sum_{i} k_{i} \varepsilon_{s_{i}}(d s)$ where $\ell$ is a bounded Borel function, $\varepsilon_{a}$ the Dirac measure at $a$, and $f(t, y, \widehat{y}, z, u)$ is Lipschitz. Since $\int_{t}^{t+\delta}\left|a_{s}\right|\left|Y_{s}\right| \eta(d s) \leq$ $K\left(\int_{t}^{t+\delta}\left|Y_{s}\right| d s+\sum_{i} \mathbb{1}_{s_{i} \in[t, t+\delta]} Y_{s_{i}}\right)$, the driver satisfies the Lipschitz condition, and the above ABSDE has a solution.

\subsection{Linear ABSDE}

In this part we give a closed formula for the solution of linear ABSDEs. That means the driver $f$ is linear w.r.t. $Y, Z$ and $U$. We first give a result about the form of $Y$, part of the solution of a linear ABSDEs in the Brownian case.

Proposition 4.1. Consider the following ABSDE

$$
\left\{\begin{aligned}
-d Y_{t} & =\left[<\overrightarrow{\mathbf{a}}_{t}, \overrightarrow{\mathbf{Y}}_{t}>+l_{t}\right] d t-Z_{t} d B_{t}, \quad 0 \leq t \leq T \\
Y_{T+t} & =\xi_{T+t}, \quad 0 \leq t \leq \delta \\
Z_{T+t} & =P_{T+t}, \quad 0<t \leq \delta
\end{aligned}\right.
$$

where $\langle\cdot, \cdot>$ is the scalar product, $\overrightarrow{\mathbf{a}}$ and $\overrightarrow{\mathbf{Y}}$ are defined by

$$
\begin{aligned}
\overrightarrow{\mathbf{a}}_{t} & =\left(\mu_{t}, \bar{\mu}_{t}, \underline{\mu}_{t}, \sigma_{t}, \bar{\sigma}_{t}, \underline{\sigma}_{t}\right) \\
\overrightarrow{\mathbf{Y}}_{t} & =\left(Y_{t}, \mathbb{E}^{\mathcal{F}_{t}}\left[p_{t+\delta} Y_{t+\delta}\right], \mathbb{E}^{\mathcal{F}_{t}}\left(\int_{0}^{\delta} p_{t+u} Y_{t+u} d u\right), Z_{t}, \mathbb{E}^{\mathcal{F}_{t}}\left[q_{t+\delta} Z_{t+\delta}\right], \mathbb{E}^{\mathcal{F}_{t}}\left(\int_{0}^{\delta} q_{t+u} Z_{t+u} d u\right]\right),
\end{aligned}
$$

and where the processes $\mu, \bar{\mu}, \underline{\mu}, \sigma, \bar{\sigma}, \underline{\sigma} \in L_{\mathbb{F}}^{2}[-\delta, T+\delta]$ are assumed to be uniformly bounded and $l \in L_{\mathbb{F}}^{2}[0, T]$. Then, for any $t \in[0, T]$, the solution $Y$ is given by

$$
\begin{aligned}
Y_{t}= & \mathbb{E}^{\mathcal{F}_{t}}\left[X_{T}^{t} \xi_{T}+\int_{t}^{T} l_{s} X_{s}^{t} d s+\int_{T}^{T+\delta}\left\{\bar{\mu}_{s-\delta} p_{s} \xi_{s}+\bar{\sigma}_{s-\delta} q_{s} P_{s}\right\} X_{s-\delta}^{t} d s\right. \\
& \left.+\int_{T}^{T+\delta} \int_{0}^{\delta}\left\{\underline{\mu}_{s-u} p_{s} \xi_{s}+\underline{\sigma}_{s-u} q_{s} P_{s}\right\} X_{s-u}^{t} d u d s\right],
\end{aligned}
$$


where

$$
\left\{\begin{aligned}
d X_{s}^{t}= & {\left[\mu_{s} X_{s}^{t}+p_{s} \bar{\mu}_{s-\delta} X_{s-\delta}^{t}+p_{s} \int_{0}^{\delta} \underline{\mu}_{s-u} X_{s-u}^{t} d u\right] d s } \\
& +\left[\sigma_{s} X_{s}^{t}+q_{s} \bar{\sigma}_{s-\delta} X_{s-\delta}^{t}+q_{s} \int_{0}^{\delta} \underline{\sigma}_{s-u} X_{s-u}^{t} d u\right] d B_{s}, \quad t \leq s \leq T+\delta, \\
X_{t}^{t}= & 1, \\
X_{s}^{t}= & 0, \quad t-\delta \leq s \leq t .
\end{aligned}\right.
$$

Proof. The proof of this result is similar to the proof of Theorem 2.1 in [12]. We give some details in Appendix.

We now extend the previous result to the case of an ABSDE with jump.

Proposition 4.2. Consider the following ABSDE

$$
\left\{\begin{array}{l}
-d Y_{t}=\left[<\overrightarrow{\mathbf{a}}_{t}, \overrightarrow{\mathbf{Y}}_{t}>+l_{t}\right] d t-Z_{t} d B_{t}-U_{t} d H_{t}, \quad 0 \leq t \leq T, \\
Y_{T+t}=\xi_{T+t}, \quad 0 \leq t \leq \delta \\
Z_{T+t}=P_{T+t}, \quad U_{T+t}=Q_{T+t} \mathbb{1}_{\{T+t \leq \tau\}}, \quad 0<t \leq \delta
\end{array}\right.
$$

where $\langle\cdot, \cdot\rangle$ is the scalar product, and $\overrightarrow{\mathbf{a}}$ and $\overrightarrow{\mathbf{Y}}$ are defined by

$$
\begin{aligned}
\overrightarrow{\mathbf{a}}_{t} & =\left(\mu_{t}, \bar{\mu}_{t}, \underline{\mu}_{t}, \sigma_{t}, \overline{\sigma_{t}}, \underline{\sigma}_{t}, \rho_{t}, \bar{\rho}_{t}, \underline{\rho}_{t}\right), \\
\overrightarrow{\mathbf{Y}}_{t} & =\left(Y_{t}, \mathbb{E}^{\mathcal{G}_{t}}\left[Y_{t+\delta}\right], \mathbb{E}^{\mathcal{G}_{t}}\left[\int_{0}^{\delta} Y_{t+u} d u\right], Z_{t}, \mathbb{E}^{\mathcal{G}_{t}}\left[Z_{t+\delta}\right], \mathbb{E}^{\mathcal{G}_{t}}\left[\int_{0}^{\delta} Z_{t+u} d u\right], U_{t}, \mathbb{E}^{\mathcal{G}_{t}}\left[U_{t+\delta}\right], \mathbb{E}^{\mathcal{G}_{t}}\left[\int_{0}^{\delta} U_{t+u} d u\right]\right) .
\end{aligned}
$$

We assume the functions $\mu, \bar{\mu}, \mu, \sigma, \bar{\sigma}, \underline{\sigma}, \rho, \bar{\rho}, \rho \in L_{\mathbb{G}}^{2}[-\delta, T+\delta]$ are assumed to be uniformly bounded and $l \in$ $L_{\mathbb{G}}^{2}[0, T]$.

Then, for any $t \in[0, T]$, the solution $Y$ is given by $Y_{t}=Y_{t}^{b} \mathbb{1}_{t<\tau}+Y_{t}^{a}(\tau) \mathbb{1}_{t \geq \tau}$ where $Y^{b}$ and $Y^{a}(\theta)$, for any $\theta \in[0, T]$, are defined by

$$
\left\{\begin{aligned}
Y_{t}^{a}(\theta)= & \mathbb{E}^{\mathcal{F}_{t}}\left[X_{T}^{t, a}(\theta) \xi_{T}^{a}(\theta)+\int_{t}^{T} X_{s}^{t, a}(\theta) l_{s}^{a}(\theta) d s+\int_{T}^{T+\delta}\left(\xi_{s}^{a}(\theta) \bar{\mu}_{s-\delta}^{a}(\theta)\right.\right. \\
& \left.\left.+P_{s}^{a}(\theta) \bar{\sigma}_{s-\delta}^{a}(\theta) X_{s-\delta}^{t, a}(\theta)\right) d s+\int_{T}^{T+\delta} \int_{0}^{\delta}\left(\xi_{s}^{a}(\theta) \underline{\mu}_{s-u}^{a}(\theta)+P_{s}^{a}(\theta) \underline{\sigma}_{s-u}^{a}(\theta)\right) X_{s-u}^{t, a}(\theta) d u d s\right] \\
Y_{t}^{b}= & \mathbb{E}^{\mathcal{F}_{t}}\left[X_{T}^{t, b} \xi_{T}^{b}+\int_{t}^{T} X_{s}^{t, b} L_{s} d s+\int_{T}^{T+\delta}\left(G_{s} \xi_{s}^{b} \bar{\mu}_{s-\delta}^{b}+G_{s} P_{s}^{b} \bar{\sigma}_{s-\delta}^{b}\right) X_{s-\delta}^{t, b} d s\right. \\
& \left.+\int_{T}^{T+\delta} \int_{0}^{\delta}\left(G_{s} \xi_{s}^{b} \bar{\mu}_{s-u}^{b}+G_{s} P_{s}^{b} \bar{\sigma}_{s-u}^{b}\right) X_{s-u}^{t, b} d u d s\right]
\end{aligned}\right.
$$

with

$$
\begin{aligned}
L_{t}= & l_{t}^{b}+\bar{\mu}_{t}^{b} J_{t}^{Y^{a}}(t+\delta)+\underline{\mu}_{t}^{b} \int_{0}^{\delta} J_{t}^{Y^{a}}(t+u) d u+\bar{\sigma}_{t}^{b} J_{t}^{Z^{a}}(t+\delta)+\underline{\sigma}_{t}^{b} \int_{0}^{\delta} J_{t}^{Z^{a}}(t+u) d u \\
& +\rho_{t}^{b} Y_{t}^{a}(t)+\frac{\bar{\rho}_{t}^{b}}{G_{t}} \mathbb{E}^{\mathcal{F}_{t}}\left[G_{t+\delta} Y_{t+\delta}^{a} \mathbb{1}_{\{t+\delta \leq T\}}+G_{t+\delta} Q_{t+\delta} \mathbb{1}_{\{t+\delta>T\}}\right] \\
& +\frac{\underline{\rho}_{t}^{b}}{G_{t}} \mathbb{E}^{\mathcal{F}_{t}}\left[\int_{0}^{\delta}\left(Y_{t+u}^{a}(t+u) \mathbb{1}_{\{t+u \leq T\}}+Q_{t+u} \mathbb{1}_{\{t+u>T\}}\right) G_{t+u} d u\right]
\end{aligned}
$$


where $X^{t, a}(\theta)$ is the solution of the following linear stochastic delayed differential equation (SDDE)

$$
\left\{\begin{aligned}
d X_{s}^{t, a}(\theta)= & {\left[\mu_{s}^{a} X_{s}^{t, a}(\theta)+\bar{\mu}_{s-\delta}(\theta) X_{s-\delta}^{t, a}(\theta)+\int_{0}^{\delta} \underline{\mu}_{s-u}^{a}(\theta) X_{s-u}^{t, a}(\theta) d u\right] d s } \\
& +\left[\sigma_{s-\delta}^{a}(\theta) X_{s}^{t, a}(\theta)+\bar{\sigma}_{s-\delta}^{a} X_{s-\delta}^{t, a}(\theta)+\int_{0}^{\delta} \underline{\sigma}_{s-u}^{a}(\theta) X_{s-u}^{t, a}(\theta) d u\right] d B_{s}, \quad s \in[t, T+\delta] \\
X_{t}^{t, a}(\theta)= & 1, \\
X_{s}^{t, a}(\theta)= & 0, \quad s \in[t-\delta, t]
\end{aligned}\right.
$$

and $X^{t, b}$ is the solution of the following linear SDDE

$$
\left\{\begin{aligned}
d X_{s}^{t, b}= & {\left[\left(\mu_{s}^{b}-\rho_{s}^{b}\right) X_{s}^{t, b}+\left(\frac{\bar{\mu}_{s-\delta}^{b}}{G_{s-\delta}}-\frac{\bar{\rho}_{s-\delta}^{b}}{G_{s-\delta}} \mathbb{1}_{\{s \leq T\}}\right) X_{s-\delta}^{t, b}\right.} \\
& \left.+\int_{0}^{\delta}\left(\frac{\underline{\mu}_{s-u}^{b}}{G_{s-u}}-\frac{\underline{\rho}_{s-u}^{b}}{G_{s-u}} \mathbb{1}_{\{s-u \leq T\}}\right) X_{s-u}^{t, b} d u\right] d s \\
& +\left[\sigma_{s}^{b} X_{s}^{t, b}+\frac{\bar{\sigma}_{s-\delta}^{b}}{G_{s-\delta}} X_{s-\delta}^{t, b}+\int_{0}^{\delta} \frac{\underline{\sigma}_{s-u}^{b}}{G_{s-u}} X_{s-u}^{t, b} d u\right] d B_{s}, \quad s \in[t, T+\delta], \\
X_{t}^{t, b}= & 1, \\
X_{s}^{t, b}= & 0, \quad s \in[t-\delta, t] .
\end{aligned}\right.
$$

Proof. The result is an application of the results of Section 2 and Proposition 4.1.

\section{Appendix}

\subsection{Proof of Proposition $\mathbf{1 . 1 0}$}

Applying Itô's formula to $e^{\beta s} Y_{s}^{2}$, we obtain for any $s \in[0, T]$

$$
e^{\beta T} \xi_{T}^{2}-e^{\beta t} Y_{t}^{2}=\int_{t}^{T} e^{\beta s}\left(\beta Y_{s}^{2}+Z_{s}^{2}\right) d s-2 \int_{t}^{T} e^{\beta s} Y_{s} f\left(s, \overrightarrow{\mathbf{Y}}_{s}\right) d s+2 \int_{t}^{T} e^{\beta s} Y_{s} Z_{s} d B_{s}
$$

where $\overrightarrow{\mathbf{Y}}_{s}=\left(Y_{s}, \mathbb{E}^{\mathcal{F}_{s}}\left[p_{s+\delta} Y_{s+\delta}\right],\left\{\mathbb{E}^{\mathcal{F}_{s}}\left[p_{s+u} Y_{s+u}\right]\right\}_{0 \leq u \leq \delta}, Z_{s}, \mathbb{E}^{\mathcal{F}_{s}}\left[q_{s+\delta} Z_{s+\delta}\right],\left\{\mathbb{E}^{\mathcal{F}_{s}}\left[q_{s+u} Z_{s+u}\right]\right\}_{0 \leq u \leq \delta}\right)$. By the Lipschitz assumption on $f$, using similar estimations as in the previous proofs and the boundedness of $p$ and $q$, we obtain there exists $K$ such that

$$
\begin{aligned}
f\left(t, \overrightarrow{\mathbf{Y}}_{t}\right) & \leq\left|f\left(t, \overrightarrow{\mathbf{Y}}_{t}\right)-f(t, \overrightarrow{\mathbf{0}})\right|+f(t, \overrightarrow{\mathbf{0}}) \\
& \leq K\left(\left|Y_{t}\right|+\left|Z_{t}\right|+\mathbb{E}^{\mathcal{F}_{t}}\left[\left|Y_{t+\delta}\right|+\left|Z_{t+\delta}\right|\right]+\mathbb{E}^{\mathcal{F}_{t}}\left[\int_{0}^{\delta}\left(\left|Y_{t+u}\right|+\left|Z_{t+u}\right|\right) d u\right]\right)+f(t, \overrightarrow{\mathbf{0}}) .
\end{aligned}
$$

Combining (5.1) and (5.2), and using several times that $2|a b| \leq \frac{a^{2}}{c}+c b^{2}$ for different positive constants $c$, as for example

$$
2\left|Y_{s}\right| \mathbb{E}^{\mathcal{F}_{s}}\left(\left|Y_{s+\delta}\right|\right) \leq \frac{Y_{s}^{2}}{c_{1}}+c_{1}\left(\mathbb{E}^{\mathcal{F}_{s}}\left(Y_{s+\delta}\right)\right)^{2}
$$


we get after some computations

$$
\begin{aligned}
& e^{\beta t}\left|Y_{t}\right|^{2}+\left[\beta-\left(2+c_{1}+c_{2}+c_{3}+c_{4}+c_{5}\right) K\right] \int_{t}^{T} e^{\beta s} Y_{s}^{2} d s+\left[1-\frac{K}{c_{3}}\right] \int_{t}^{T} e^{\beta s} Z_{s}^{2} d s \\
\leq & e^{\beta T} \xi_{T}^{2}-2 \int_{t}^{T} e^{\beta s} Y_{s} Z_{s} d B_{s}+2 \int_{t}^{T} e^{\beta s} Y_{s} f(s, \overrightarrow{\mathbf{0}}) d s+\frac{K}{c_{1}} \int_{t}^{T} e^{\beta s} \mathbb{E}^{\mathcal{F}_{s}}\left(Y_{s+\delta}^{2}\right) d s \\
& +\frac{K}{c_{2}} \int_{t}^{T} e^{\beta s} \int_{0}^{\delta} \mathbb{E}^{\mathcal{F}_{s}}\left(Y_{s+u}^{2}\right) d u d s+\frac{K}{c_{4}} \int_{t}^{T} e^{\beta s} \mathbb{E}^{\mathcal{F}_{s}}\left(Z_{s+\delta}^{2}\right) d s+\frac{K}{c_{5}} \int_{t}^{T} e^{\beta s} \int_{0}^{\delta} \mathbb{E}^{\mathcal{F}_{s}}\left(Z_{s+u}^{2}\right) d u d s
\end{aligned}
$$

Taking conditional expectation w.r.t. $\mathcal{F}_{t}$, noting that from Fubini

$$
\int_{t}^{T} e^{\beta s} \int_{0}^{\delta} Y_{s+u}^{2} d u d s \leq \delta \int_{t}^{T+\delta} e^{\beta s} Y_{s}^{2} d s
$$

and that

$$
\int_{t}^{T} e^{\beta s} Y_{s+\delta}^{2} d s \leq \int_{t}^{T+\delta} e^{\beta s} Y_{s}^{2} d s
$$

we obtain, after some simplifications, and using again $2|a b| \leq \frac{a^{2}}{c}+c b^{2}$ (with a new constant $c_{6}$ !)

$$
\begin{gathered}
e^{\beta t}\left|Y_{t}\right|^{2}+\left[\beta-\left(2+c_{1}+c_{2}+c_{3}+c_{4}+c_{5}\right) K-\frac{K}{c_{1}}-\frac{\delta K}{c_{2}} K-c_{6}\right] \mathbb{E}^{\mathcal{F}_{t}}\left(\int_{t}^{T} e^{\beta s} Y_{s}^{2} d s\right) \\
+\left[1-\frac{K}{c_{3}}-\frac{K}{c_{4}}-\frac{\delta K}{c_{5}}\right] \mathbb{E}^{\mathcal{F}_{t}}\left(\int_{t}^{T} e^{\beta s} Z_{s}^{2} d s\right) \\
\leq e^{\beta T} \mathbb{E}^{\mathcal{F}_{t}}\left(\xi_{T}^{2}\right)+\frac{1}{c_{6}} \mathbb{E}^{\mathcal{F}_{t}}\left(\int_{t}^{T} e^{\beta s}(f(s, \overrightarrow{\mathbf{0}}))^{2} d s\right)+\left[\frac{\delta K}{c_{2}}+\frac{K}{c_{1}}\right] \mathbb{E}^{\mathcal{F}_{t}}\left(\int_{T}^{T+\delta} \xi_{s}^{2} d s\right) \\
+\left[\frac{\delta K}{c_{5}}+\frac{K}{c_{4}}\right] \mathbb{E}^{\mathcal{F}_{t}}\left(\int_{T}^{T+\delta} P_{s}^{2} d s\right) .
\end{gathered}
$$

It remains to choose the constants $c_{i}$ such that $\beta=\left(2+c_{1}+c_{2}+c_{3}+c_{4}+c_{5}\right) K+\frac{K}{c_{1}}+\frac{\delta K}{c_{2}} K+c_{6}$ and $\frac{K}{c_{3}}+\frac{K}{c_{4}}+\frac{\delta K}{c_{5}} \leq 1$ to obtain that there exists a constant $K$ such that

$$
\mathbb{E}^{\mathcal{F}_{t}}\left[\int_{t}^{T} Z_{s}^{2} d s\right] \leq K \mathbb{E}^{\mathcal{F}_{t}}\left[\xi_{T}^{2}+\int_{T}^{T+\delta}\left(\xi_{s}^{2}+P_{s}^{2}\right) d s+\int_{t}^{T}(f(s, \overrightarrow{\mathbf{0}}))^{2} d s\right]
$$

for any $t \in[0, T]$.

The proof for the term with the supremum on $Y$ is obtained using the same methodology and BurkholderDavis-Gundy inequality. 


\subsection{Proof of Proposition 4.1}

Applying Itô's formula to $X^{t} Y$ and taking the conditional expectation we get

$$
\begin{aligned}
X_{t}^{t} Y_{t}= & \mathbb{E}^{\mathcal{F}_{t}}\left[X_{T}^{t} Y_{T}+\int_{t}^{T} l_{s} X_{s}^{t} d s+\int_{t}^{T} \bar{\mu}_{s} X_{s}^{t} p_{s+\delta} Y_{s+\delta} d s-\int_{t}^{T} \bar{\mu}_{s-\delta} X_{s-\delta}^{t} p_{s} Y_{s} d s\right. \\
& +\int_{t}^{T} \bar{\sigma}_{s} X_{s}^{t} q_{s+\delta} Z_{s+\delta} d s-\int_{t}^{T} \bar{\sigma}_{s-\delta} X_{s-\delta}^{t} q_{s} Z_{s} d s \\
& +\int_{t}^{T} \underline{\mu}_{s} X_{s}^{t} \int_{0}^{\delta} p_{s+u} Y_{s+u} d u d s-\int_{t}^{T} p_{s} Y_{s} \int_{0}^{\delta} \underline{\mu}_{s-u} X_{s-u}^{t} d u d s \\
& \left.+\int_{t}^{T} \underline{\sigma}_{s} X_{s}^{t} \int_{0}^{\delta} q_{s+u} Z_{s+u} d u d s-\int_{t}^{T} q_{s} Z_{s} \int_{0}^{\delta} \underline{\sigma}_{s-u} X_{s-u}^{t} d u d s\right]
\end{aligned}
$$

This can be rewritten, by using $X_{u}^{t}=0$ for $u \leq t$, under the form

$$
\begin{aligned}
X_{t}^{t} Y_{t}= & \mathbb{E}^{\mathcal{F}_{t}}\left[X_{T}^{t} Y_{T}+\int_{t}^{T} l_{s} X_{s}^{t} d s+\int_{t}^{T+\delta} \bar{\mu}_{s-\delta} X_{s-\delta}^{t} p_{s} Y_{s} d s-\int_{t}^{T} \bar{\mu}_{s-\delta} X_{s-\delta}^{t} p_{s} Y_{s} d s\right. \\
& +\int_{t}^{T+\delta} \bar{\sigma}_{s-\delta} X_{s-\delta}^{t} q_{s} Z_{s} d s-\int_{t}^{T} \bar{\sigma}_{s-\delta} X_{s-\delta}^{t} q_{s} Z_{s} d s+\int_{t}^{T+\delta} \int_{0}^{\delta} \underline{\mu}_{s-u} X_{s-u}^{t} p_{s} Y_{s} d u d s \\
& -\int_{t}^{T} \int_{0}^{\delta} \underline{\mu}_{s-u} X_{s-u}^{t} p_{s} Y_{s} d u d s+\int_{t}^{T+\delta} \int_{0}^{\delta} \underline{\sigma}_{s-u} X_{s-u}^{t} q_{s} Z_{s} d u d s \\
& \left.-\int_{t}^{T} \int_{0}^{\delta} \underline{\sigma}_{s-u} X_{s-u}^{t} q_{s} Z_{s} d u d s\right] .
\end{aligned}
$$

Since $X_{t}^{t}=1$, we conclude

$$
\begin{aligned}
Y_{t}= & \mathbb{E}^{\mathcal{F}_{t}}\left[X_{T}^{t} \xi_{T}+\int_{t}^{T} l_{s} X_{s}^{t} d s+\int_{T}^{T+\delta}\left\{\bar{\mu}_{s-\delta} p_{s} \xi_{s}+\bar{\sigma}_{s-\delta} q_{s} P_{s}\right\} X_{s-\delta}^{t} d s\right. \\
& \left.+\int_{T}^{T+\delta} \int_{0}^{\delta}\left\{\underline{\mu}_{s-u} p_{s} \xi_{s}+\underline{\sigma}_{s-u} q_{s} P_{s}\right\} X_{s-u}^{t} d u d s\right] .
\end{aligned}
$$

\subsection{Without Immersion property}

We present how to solve the study of ABSDE in a general setting, without immersion hypothesis and explain that it will only require more attention and more complex computations.

A first method is to note that, under density Hypothesis 1.2, it is known that $B$ is a $\mathbb{G}$-semimartingale of the form

$$
B_{t}=B_{t}^{\mathbb{G}}+\int_{0}^{t \wedge \tau} \frac{d\langle B, G\rangle_{s}^{\mathbb{F}}}{G_{s-}}+\int_{t \wedge \tau}^{t} \frac{\left.d\langle B, \alpha(u)\rangle_{s}^{\mathbb{F}}\right|_{u=\tau}}{\alpha_{s}(\tau)}=: B_{t}^{\mathbb{G}}+\int_{0}^{t} \nu_{s} d s
$$

where $G_{t}=\mathbb{P}\left(\tau>t \mid \mathcal{F}_{t}\right)$ is now a supermartingale and $B^{\mathbb{G}}$ is a $\mathbb{G}$-Brownian motion. Therefore, the BSDE (0.1) has the form

$$
\left\{\begin{aligned}
-d Y_{t}= & \left(f \left(t, Y_{t}, \mathbb{E}^{\mathcal{G}_{t}}\left[Y_{t+\delta}\right],\left(\mathbb{E}^{\mathcal{G}_{t}}\left[Y_{t+s}\right]\right)_{0 \leq s \leq \delta}, Z_{t}, \mathbb{E}^{\mathcal{G}_{t}}\left[Z_{t+\delta}\right],\left(\mathbb{E}^{\mathcal{G}_{t}}\left[Z_{t+s}\right]\right)_{0 \leq s \leq \delta},\right.\right. \\
& \left.\left.U_{t}, \mathbb{E}^{\mathcal{G}_{t}}\left[U_{t+\delta}\right],\left(\mathbb{E}^{\mathcal{G}_{t}}\left[U_{t+s}\right]\right)_{0 \leq s \leq \delta}\right)+Z_{t} \nu_{t}\right) d t-Z_{t} d B_{t}^{\mathbb{G}}-U_{t} d H_{t},
\end{aligned}\right.
$$


and where $Y_{T}$ is given, and the same analysis can be done, under a square integrability assumption on $\nu$, which can be justified in a financial meaning to avoid arbitrages in the enlarged filtration.

A second method ${ }^{2}$ is based on the change of probability on the initial enlarged filtration suggested in the Introduction, i.e.,

$$
\left.d \mathbb{P}^{*}\right|_{\mathcal{F}_{t} \vee \sigma(\tau)}=\left.\ell_{t} d \mathbb{P}\right|_{\mathcal{F}_{t} \vee \sigma(\tau)},
$$

has to be used on $\mathcal{G}_{t}$ and is

$$
\left.d \mathbb{P}^{*}\right|_{\mathcal{G}_{t}}=\left.\ell_{t} d \mathbb{P}\right|_{\mathcal{G}_{t}}
$$

where $\ell$ is given by

$$
\ell_{t}=\mathbb{E}\left(\left(\alpha_{t}(\tau)\right)^{-1} \mid \mathcal{G}_{t}\right)=\mathbb{1}_{t<\tau} \frac{G(t)}{G_{t}}+\mathbb{1}_{\tau \leq t} \frac{1}{\alpha_{t}(\tau)},
$$

with $G(t)=\mathbb{P}(\tau>t)$. Under $\mathbb{P}^{*}$, the random time $\tau$ is independent of $\mathcal{F}_{t}$ and $B$ is a $\mathbb{P}^{*}$-Brownian motion. Under that probability, the ABSDE (0.1) has the form

$$
\left\{\begin{aligned}
-d Y_{t}= & f\left(t, Y_{t}, \ell_{t} \mathbb{E}^{*} \mathcal{G}_{t}\left[\left(\ell_{t+\delta}\right)^{-1} Y_{t+\delta}\right], \ell_{t}\left(\mathbb{E}^{* \mathcal{G}_{t}}\left[\left(\ell_{t+s}\right)^{-1} Y_{t+s}\right]\right)_{0 \leq s \leq \delta}, Z_{t}, \ell_{t} \mathbb{E}^{* \mathcal{G}_{t}}\left[\left(\ell_{t+\delta}\right)^{-1} Z_{t+\delta}\right],\right. \\
& \left.\ell_{t}\left(\mathbb{E}^{*} \mathcal{G}_{t}\left[\left(\ell_{t+s}\right)^{-1} Z_{t+s}\right]\right)_{0 \leq s \leq \delta}, U_{t}, \ell_{t} \mathbb{E}^{* \mathcal{G}_{t}}\left[\left(\ell_{t+\delta}\right)^{-1} U_{t+\delta}\right], \ell_{t}\left(\mathbb{E}^{* \mathcal{G}_{t}}\left[\left(\ell_{t+s}\right)^{-1} U_{t+s}\right]\right)_{0 \leq s \leq \delta}\right) d t \\
& -Z_{t} d B_{t}-U_{t} d H_{t},
\end{aligned}\right.
$$

and where $Y_{T}$ is given. The same methodology works, providing conditions on $\ell$ such that the required Lipschitz conditions are satisfied.

Aknowledgement: We thank the anonymous referee for his (her) comments.

\section{REFERENCES}

[1] A. Aksamit, and M. Jeanblanc, Enlargement of filtration with finance in view, Springer, forthcoming.

[2] P. Brémaud, and M. Yor, Changes of filtration and of probability measures, Z. Wahr. Verw. Gebiete, 45, 269-295, 1978.

[3] R. Dumitrescu, M-C. Quenez, and A. Sulem, BSDEs with default jump, Preprint, 2016.

[4] N. El Karoui, M. Jeanblanc, and Y. Jiao, What happens after a default: the conditional density approach, Stochastic Processes and their Appl., 120(7), 1011-1032, 2010.

[5] N. El Karoui, S. Peng, and M-C. Quenez, Backward stochastic differential equations in finance, Mathematical Finance, 7 (1), $1-71,1997$.

[6] T. Jeulin and M. Yor, Grossissement de filtration: exemples et applications, Lecture Notes in Maths, 1118, Springer, 1985.

[7] T. Jeulin, Semimartingales et grossissements d'une filtration, Lecture Notes in Maths, 833, Springer, 1980.

[8] I. Kharroubi and T. Lim, Progressive enlargement of filtrations and backward stochastic differential equations with jumps, Journal of Theoretical Probability, 27 (3), 683-724, 2014.

[9] I. Kharroubi, T. Lim and A. Ngoupeyou, Mean-variance hedging on uncertain time horizon in a Market with a Jump, Applied Mathematics and Optimization, 68 (3), 413-444, 2013.

[10] A. Ngoupeyou, Optimisation des portefeuilles d'actifs soumis au risque de défaut, PHD Thesis Evry university 2010.

[11] B. Øksendal, A. Sulem, and T. Zhang, Optimal control of stochastic delay equations and time-advanced backward stochastic differential equations, Adv. Appl. Prob., 43, 572-596, 2011.

[12] S. Peng and Z. Yang, Anticipated backward stochastic differential equations, The Annals of Probability, 37 (3), 877-902, 2009.

[13] H. Pham, Stochastic control under progressive enlargement of filtrations and applications to multiple defaults risk management, Stochastic Processes and their Applications, 120(9), 1795-1820, 2010.

[14] S. Song, Optional splitting formula in a progressively enlarged filtration, ESAIM Probability and Statistics, 18, 829-853, 2014.

\footnotetext{
$2^{2}$ suggested by the anonymous referee
} 